\title{
二芳基甲酮亚胺的不对称催化氢化硅烷化反应研究
}

\author{
扈晓艳 ${ }^{a, b}$ 胡方芝 ${ }^{a, b}$ 张敏敏 ${ }^{a, b}$ 廖益均 ${ }^{a, b}$ 徐小英 ${ }^{a}$ \\ 袁伟成 ${ }^{a}$ 张晓梅*, ${ }^{*}$ \\ ( ${ }^{a}$ 中国科学院成都有机化学研究所 成都 610041) \\ ${ }^{b}$ 中国科学院大学 北京 100049)
}

\begin{abstract}
摘要 发展了手性路易斯碱催化的 2-位无取代基的二芳基甲酮亚胺的不对称氢化硅烷化反应, 在 $20 \mathrm{~mol} \%$ 的最优催化 剂催化下，以良好的收率(最高达 97\%)和中等到良好的对映选择性(最高达 $89 \%$ )获得了一系列手性二芳基甲胺类化合 物，并通过单晶 $\mathrm{X}$ 射线衍射的方法确定了其中一个反应产物的绝对构型.

关键词 二芳基甲胺; 二芳基甲酮亚胺; 手性路易斯碱; 氢化硅烷化
\end{abstract}

\section{Enantioselective Hydrosilylation of $\mathrm{N}$-Aryl Diaryl Ketimines}

\author{
Hu, Xiaoyan ${ }^{a, b}$ \\ Hu, Fangzhi ${ }^{a, b}$ \\ Zhang, Minmin ${ }^{a, b}$ \\ Liao, Yijun ${ }^{a, b}$ \\ $\mathrm{Xu}$, Xiaoying ${ }^{a, b}$ \\ Yuan, Weicheng ${ }^{a}$ \\ Zhang, Xiaomei*,a \\ ( ${ }^{a}$ Chengdu Institute of Organic Chemistry, Chinese Academy of Sciences, Chengdu, 610041) \\ ( ${ }^{b}$ University of Chinese Academy of Sciences, Beijing, 100049)
}

\begin{abstract}
Lewis base catalyzed enantioselective hydrosilylation of non-ortho-substituted $N$-aryl diaryl ketimines was realized. In the presence of $20 \mathrm{~mol} \%$ of the optimal catalyst, the reactions provided a series of (diarylmethyl)amines with high yields (up to $97 \%$ ) in moderate to good enantioselectivities (up to $89 \%$ ee). The absolute configuration of one product was determined by X-ray crystallographic analysis.
\end{abstract}

Keywords diarylmethylamines; diaryl ketimines; chiral Lewis base; hydrosilylation

手性二芳基甲胺是一类非常重要的化合物, 许多手 性药物和生理活性分子中就含有手性二芳基甲胺片断, 如手性药物西替利嗪等 ${ }^{\left[{ }^{1}\right.}$. 因此, 手性二芳基甲胺的不 对称合成引起了广泛的关注, 近年来陆续有多个研究小 组进行了相关的研究, 并取得了显著的进展 ${ }^{[2]}$. 到目前 为止, 手性二芳基甲胺的不对称合成主要通过芳基亚胺 的不对称芳基加成反应来实现, 包括手性辅助基团控制 的反应 ${ }^{[3]}$ 和催化不对称加成反应 ${ }^{[4]}$. 另外有为数不多的 关于二芳基甲酮亚胺的不对称还原反应的报道 ${ }^{[5]}$. 最近, 于金泉课题组 ${ }^{[6]}$ 研究了钯催化的对称二芳基甲胺的不对 称碳氢活化碘化反应, 高对映选择性地得到了一系列 2碘代二芳基甲胺.

亚胺的不对称还原是合成手性含氮化合物的重要

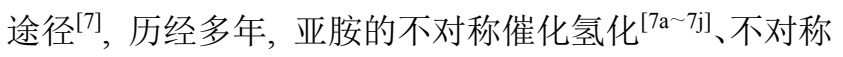

催化氢转移反应 ${ }^{[7 \mathrm{k} \sim 7 \mathrm{p}]}$ 以及不对称催化氢化硅烷化反 应 $^{[7 \mathrm{q} \sim 7 \mathrm{~s}]}$ 等已得到了长足的发展, 各种类型亚胺的不对 称催化还原取得了很高的活性和对映选择性, 但是, 关 于二芳基甲酮亚胺的不对称还原反应的报道还很少 ${ }^{[5]}$. 在二芳基甲酮亚胺的不对称还原反应的研究中, 目前大 部分的底物都是在其中一个芳基的 2-位带有取代基, 这 样在两个芳基之间形成立体效应的差别，从而获得高对 映选择性，而芳基的 2-位没有取代基的底物通常只能得 到很低的对映选择性. 2013 年 Cabrera 等 ${ }^{[5 e}$ 报道了八羰 基合钴与 $( \pm)-2,2^{\prime}$-双 (二苯膦基)-1,1'-联萗(BINAP)的配 合物催化的二芳基甲酮亚胺的不对称氢化反应，该体系 对于芳基 2-位没有取代基的二芳基甲酮亚胺取得了优 良的对映选择性, 这是迄今为止唯一一例相关的报道.

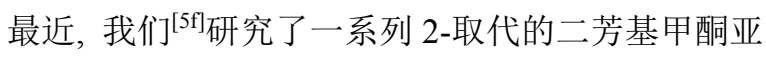

\footnotetext{
* E-mail: xmzhang@cioc.ac.cn

Received December 30, 2015; revised April 2, 2016; published online April 7, 2016.

Project supported by the National Natural Science Foundation of China (No. 21172217).

国家自然科学基金(No. 21172217)资助项目.
} 
胺在手性路易斯碱催化下的不对称氢化硅烷化反应, 发 现反应的对映选择性除了受到立体效应的影响，反应底 物的电子效应也非常明显. 如果去除 2-位取代基, 完全 排除立体因素的影响, 单纯靠二芳基甲酮亚胺的两个芳 基之间电子云密度的差别是否也能达到高对映选择性 呢? 为此, 我们设计了 $N$-芳基-二芳基甲酮亚胺 $\mathbf{1}$, 其中 $\mathrm{Ar}^{1}$ 和 $\mathrm{Ar}^{2}$ 分别为富电子的芳基和贫电子芳基, 期望凭借 两个芳基的电子云密度的差异, 利用手性路易斯碱催化 的氢化硅烷化反应来获得光学活性的二芳基甲胺类化 合物(Eq. 1).

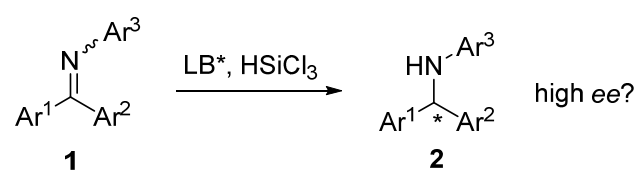

\section{1 结果与讨论}

首先, 以两个芳基分别为苯基和 2-噻吩基的亚胺 $1 \mathbf{a}$ 为标准底物, 以 1,2 -二氯乙烷为溶剂, 加入 1 equiv. 的醋酸作为添加剂, 在 $0{ }^{\circ} \mathrm{C}$ 下考查了各种吡啶甲酰胺类 手性路易斯碱催化剂(10 mol\%)对反应的影响，实验结 果见表 1 . 从表 1 可看出, 麻黄碱衍生的催化剂 $\mathbf{3 a}^{[8]}$ 可以 很好地催化该反应，得到几乎定量的收率和中等 $(67 \%)$ 的对映选择性(表 1, Entry 1), 氯霉素中间体衍生的催化 剂 $3 \mathbf{b}^{[9]}$ 得到了相似的结果(表 1 , Entry 2). 当采用丝氨酸 衍生的催化剂 $3 \mathrm{c}^{[10]}$ 时, 反应的对映选择性略微上升, 达 到了 70\%(表 1, Entry 3), 而反式差弪脯氨酸衍生的催化剂 $\mathbf{3 d}^{[11]}$ 只能得到痕量的产物(表 1, Entry 4), 可能是因为空 间位阻太大的原因, 脯氨酸衍生的催化剂 $3 \mathrm{e}^{[12]}$ 得到较 低的对映选择性(表 1, Entry 5), 因此, 3c 被确定为最佳 催化剂。

接着考察了溶剂对反应的影响, 在二氯甲烷中反应 得到了较高的对映选择性(表 1, Entry 6), 但在氯仿中反 应很困难, 只得到痕量的产物(表 1, Entry 7), 以甲苯和 四氢呋喃为溶剂, 反应活性和对映选择性都明显降低 (表 1, Entries 8,9), 因此, 二氯甲烷被确定为最佳溶剂. 为了提高对映选择性, 又尝试增加催化剂的用量, 当催 化剂用量增加到 $15 \mathrm{~mol} \%$ 时，对映选择性稍有提高(表 1, Entry 10), 当催化剂用量增加到 $20 \mathrm{~mol} \%$ 时, 对映选择 性达到 74\%(表 1, Entry 11), 但是继续增加催化剂用量 对映选择性并没有继续提高(表 1 , Entry 12), 因此, 反应 的最佳催化剂用量确定为 $20 \mathrm{~mol} \%$.

在最佳反应条件确定后, 对反应的底物普适性进行 了考察(表 2), 从表 2 可看出反应底物的电子效应对反应 结果有显著的影响. 首先, 将 2-㗌吩基团变为 2-呋喃基, 发现对映选择性显著降低(表 2, Entry 2). 然后在苯环上
表 1 反应条件的优化 ${ }^{a}$

Table 1 Optimization of Conditions<smiles>N=C(N=P)c1cccs1</smiles>

1a<smiles>C[C@H]([C@H](O)c1ccccc1)N(C)C(=O)c1ccccn1</smiles>

3a

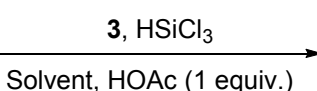
$0{ }^{\circ} \mathrm{C}, 48 \mathrm{~h}$<smiles>O=C(N[C@H]1COCO[C@H]1c1ccc([N+](=O)[O-])cc1)c1ccccn1</smiles>

3b<smiles>N=C(N)NC(c1ccccc1)c1cccs1</smiles>

$2 a$<smiles>O=C(N[C@@H]1COCO[C@]1(c1ccccc1)c1ccccn1)c1ccccc1</smiles>

3c

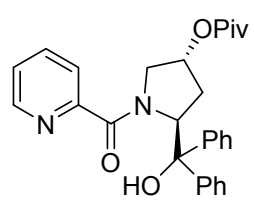

$3 d$

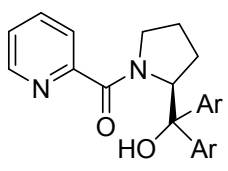

$3 e$ $\mathrm{Ar}=3,5-\mathrm{Me}_{2} \mathrm{C}_{6} \mathrm{H}_{3}$

\begin{tabular}{cclcr}
\hline Entry & Cat.* (equiv.) & \multicolumn{1}{c}{ Solvent } & Yield $^{b} \%$ & $e e^{c} / \%$ \\
\hline 1 & 3a $(0.10)$ & $\mathrm{ClCH}_{2} \mathrm{CH}_{2} \mathrm{Cl}$ & 97 & 67 \\
2 & 3b $(0.10)$ & $\mathrm{ClCH}_{2} \mathrm{CH}_{2} \mathrm{Cl}$ & 97 & -65 \\
3 & 3c $(0.10)$ & $\mathrm{ClCH}_{2} \mathrm{CH}_{2} \mathrm{Cl}$ & 97 & 70 \\
4 & 3d $(0.10)$ & $\mathrm{ClCH}_{2} \mathrm{CH}_{2} \mathrm{Cl}$ & Trace & - \\
5 & 3e $(0.10)$ & $\mathrm{ClCH}_{2} \mathrm{CH}_{2} \mathrm{Cl}$ & 97 & -58 \\
6 & 3c $(0.10)$ & $\mathrm{CH}_{2} \mathrm{Cl}_{2}$ & 97 & 72 \\
7 & 3c $(0.10)$ & $\mathrm{CHCl}_{3}$ & Trace & - \\
8 & 3c $(0.10)$ & $\mathrm{Toluene}_{2}$ & 84 & 59 \\
9 & 3c $(0.10)$ & $\mathrm{THF}$ & 90 & 63 \\
10 & 3c $(0.15)$ & $\mathrm{CH}_{2} \mathrm{Cl}_{2}$ & 97 & 73 \\
11 & 3c $(0.20)$ & $\mathrm{CH}_{2} \mathrm{Cl}_{2}$ & 97 & 74 \\
12 & 3c $(0.30)$ & $\mathrm{CH}_{2} \mathrm{Cl}_{2}$ & 97 & 72 \\
\hline
\end{tabular}

${ }^{a}$ Unless specified otherwise, the reaction was carried out with $\mathbf{1 a}(0.2 \mathrm{mmol})$, trichlorosilane $(0.8 \mathrm{mmol})$ and catalyst 3 in $2 \mathrm{~mL}$ of dichloroethane with 0.2 mmol of acetic acid as additive at $0{ }^{\circ} \mathrm{C}$ for $48 \mathrm{~h} ;{ }^{b}$ Isolated yield; ${ }^{c}$ The $e e$ values were determined by chiral HPLC.

引入各种取代基，考察了不同取代基对反应结果的影 响, 发现在苯环对位有吸电子取代基的底物通常都能取 得较好的对映选择性(表 2, Entries 3, 6, 8 10), 其中对 位硝基的底物 1i 获得了高达 83\%的对映选择性(表 2, Entry 9), 只有对位氯代的底物 1e 的对映选择性稍低(表 2, Entry 5), 说明对位吸电子取代基使得苯环更为缺电 子, 从而增大了和富电子的噻吩环的电子云密度的差 别, 因此通常能够取得较为理想的对映选择性. 而间位 有吸电子取代基的底物的反应对映选择性相对低一些, 如间位氟取代的底物 1d(表 2, Entry 4)相对于对位氟取 代的底物 $\mathbf{1 c}$ (表 2, Entry 3)，间位有两个溴取代的底物 $\mathbf{1 g}$ (表 2, Entry 7)相对于对位澳取代的底物 1f(表 2, Entry 6). 苯环对位有给电子取代基的底物则表现出对反应的 不良影响，其中对位甲氧基取代的底物 $1 \mathrm{k}$ 没有得到相 
应的产物(表 2, Entry 9), 可能是因为亚胺的两个芳基同 为富电子基团, 强给电子效应削弱了亚胺的亲电活性, 造成 $1 \mathbf{k}$ 完全没有反应活性. 苯环对位有弱给电子基甲 基的底物 $1 \mathrm{n}$ 虽然有着良好的反应活性，但对映选择性 较低(表 2, Entry 14), 而间位有给电子的甲氧基取代的 底物 11 同时表现出良好的反应活性和较好的对映选择 性(表 2, Entry 12), 说明间位的给电子效应没有对位的 给电子效应强. 值得注意的是, 在苯环的对位和间位同 时有给电子烷氧基的底物 $\mathbf{1 m}$ 虽然具有很好的反应活 性, 但却没有对映选择性(表 2, Entry 13). 此外, 在噻吩 的 5-位引入甲基使得对映选择性明显降低(表 2, Entry 15), 但在噻吩的 4-位引入甲基对反应结果没有不良影 响(表 2, Entry 16). 还考察了两个亚胺 $1 \mathbf{q}$ 和 $1 \mathbf{r}$, 它们的
两个苯基对位分别带有给电子取代基和吸电子取代基, 发现它们的对映选择性都较低(表 2, Entries 17，18). 改 变了亚胺 $\mathrm{N}$ 上的取代基，发现当 $\mathrm{N}$ 上的取代基变为苯基 时，对映选择性明显提高，达到了 89\%(表 2, Entry 19 vs. Entry 9); 当 $\mathrm{N}$ 上的取代基变为邻甲氧基苯基时, 对映选 择性则明显降低(表 2, Entry 20 vs. Entry 9), 可能是由于 邻位取代基造成亚胺构型扭曲，从而影响了反应的对映 选择性; 当 $\mathrm{N}$ 上的取代基变为对氟苯基时，在 $20 \mathrm{~mol} \%$ 催化剂作用下，只能得到痕量产物(表 2, Entry 21), 于是 我们将催化剂用量增加到 $40 \mathrm{~mol} \%$, 此时反应可以顺利 进行, 得到理想的收率和高达 $89 \%$ 的对映选择性(表 2 , Entry 22).

另外我们可看出, 反应底物的顺反比例对反应的收

表 2 反应底物范围 ${ }^{a}$

Table 2 Substrate scope

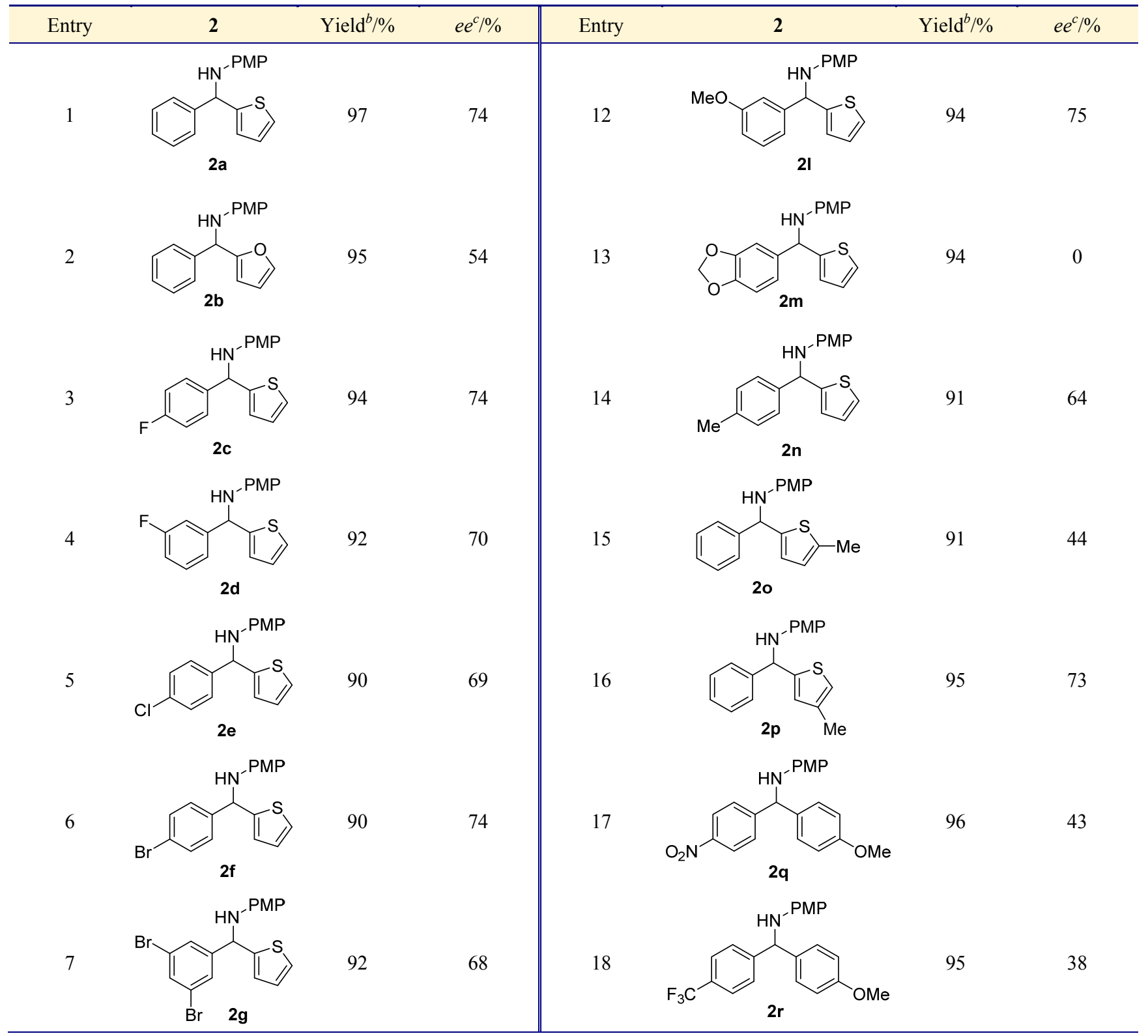




\begin{tabular}{|c|c|c|c|c|c|c|c|}
\hline Entry & 2 & Yield $^{b} / \%$ & $e e^{c} / \%$ & Entry & 2 & Yield $^{b} / \%$ & $e e^{c} / \%$ \\
\hline 8 & 2 & 93 & 72 & 19 & 2 & 87 & 89 \\
\hline 9 & & 95 & 83 & 20 & & 98 & 79 \\
\hline 10 & & 96 & 78 & 21 & $2 t$ & Trace & - \\
\hline 11 & & 0 & - & $22^{e}$ & & 93 & 89 \\
\hline
\end{tabular}

${ }^{a}$ Unless specified otherwise, the reaction was carried out with $1(0.2 \mathrm{mmol})$, trichlorosilane $(0.8 \mathrm{mmol})$ and catalyst $3 \mathbf{c}(0.04 \mathrm{mmol})$ in $2 \mathrm{~mL}$ of DCM with glacial acetic acid ( $0.2 \mathrm{mmol})$ at $0{ }^{\circ} \mathrm{C}$ for $48 \mathrm{~h} ;{ }^{b}$ Isolated yield; ${ }^{c}$ The $e e$ values were determined by chiral HPLC; ${ }^{d}$ The reactions proceeded for $24 \mathrm{~h} .{ }^{e}$ The catalyst loading is $40 \mathrm{~mol} \%$.

率和对映选择性没有影响, 这是因为亚胺在溶液中随时 都处于顺反互变中.

为了确定产物的绝对构型，我们培养了化合物 2a 的单晶, 通过 $\mathrm{X}$ 射线衍射分析确定了它的绝对构型为 $(R)\left(\right.$ 图 1) ${ }^{[13]}$.

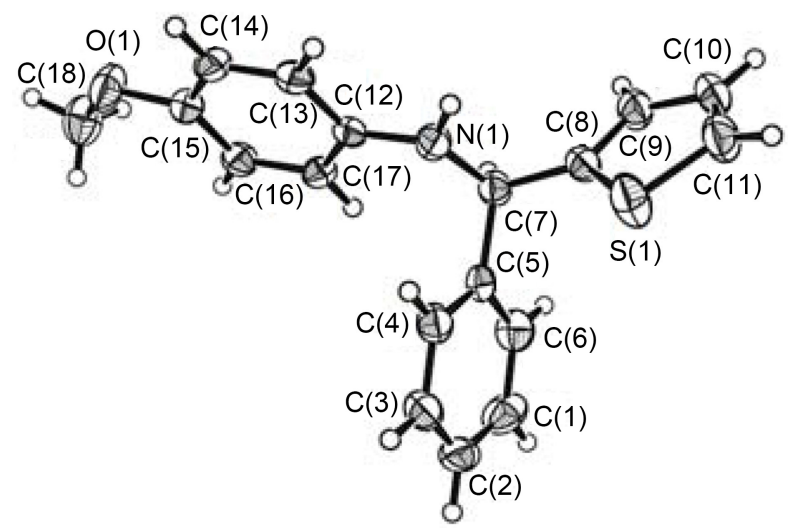

图 1 反应产物 $(R)-\mathbf{2} \mathbf{a}$ 的单晶结构

Figure 1 X-ray crystal structure of product $(R)$-2a

\section{2 结论}

我们研究了一类无 2-位取代基的二芳基甲酮亚胺
的不对称氢化硅烷化反应，在 $20 \mathrm{~mol} \%$ 的手性路易斯碱 催化剂 3c 作用下，以良好的收率和中等到良好的对映 选择性得到了一系列二芳基甲胺衍生物. 值得注意的 是，该反应完全靠底物的两个芳基的电子效应的差别获 得对映选择性. 最后, 我们通过单晶 $\mathrm{X}$ 射线衍射的方法 确定了其中一个反应产物的绝对构型.

\section{3 实验部分}

\section{1 仪器与试剂}

NMR 用 Bruker(300 MHz) 型核磁共振仪测定 $\left(\mathrm{CDCl}_{3}, \mathrm{TMS}\right.$ 为内标 $) ; \mathrm{ESI}-\mathrm{HRMS}$ 用 BioTOF $\mathrm{Q}$ 质谱仪 测定; 高效液相色谱用 Waters-Breeze 高效液相色谱仪 测定, 手性柱为 Chiralpak AD、Chiralcel OD 和 Chiralcel OJ 柱; 比旋光度用 Perkin-Elmer 341 旋光仪测定; 试剂 均为分析纯.

\section{2 实验方法}

\section{2 .1 二芳基甲酮亚胺 $\mathbf{1} \mathbf{a} \sim \mathbf{1} \mathbf{u}$ 的合成}

二芳基甲酮亚胺 $1 \mathrm{a} \sim 1 \mathrm{u}$ 按文献方法 ${ }^{[5]}$ 合成: 氮气 保护下, 将二芳基甲酮 ${ }^{[14]}(10 \mathrm{mmol})$ 、对甲氧基苯胺(3.7 $\mathrm{g}, 30 \mathrm{mmol})$ 和三乙胺 $(4.3 \mathrm{~mL}, 30 \mathrm{mmol})$ 溶于 $50 \mathrm{~mL}$ 二氯 
甲烷, 冰水浴冷却, 以注射器缓慢滴入四氯化钛 (1.0 $\mathrm{mL}, 10 \mathrm{mmol}$ ), 搅拌反应 $1 \mathrm{~h}$ 后, 撤去冰水浴, 升温至 $45{ }^{\circ} \mathrm{C}$, 继续反应 $6 \mathrm{~h}$. 旋转蒸发出去溶剂, 残余物经柱 层析和重结晶得到相应的二芳基甲酮亚胺 $\mathbf{1 a} \sim \mathbf{1 u}$.

4-甲氧基- $N$-[苯基(噻吩-2-基)亚甲基]苯胺(1a): 黄 色固体. m.p. 115.3 117.6 ${ }^{\circ} \mathrm{C} ;{ }^{1} \mathrm{H}$ NMR $(300 \mathrm{MHz}$, $\mathrm{CDCl}_{3}$, 为 $E / Z$ 约 $8 / 1$ 的混合物, 少量组分的核磁数据以 星号进行标记) $\delta: 3.71(\mathrm{~s}, 3 \mathrm{H}), 3.79 *(\mathrm{~s}, 0.4 \mathrm{H}), 6.6 \sim 6.70$ (m, 4H), 6.81* (dd, $J=19.7,8.8 \mathrm{~Hz}, 0.5 \mathrm{H}), 6.93 \sim 6.95 *$ $(\mathrm{m}, 0.2 \mathrm{H}), 6.99 \sim 7.03(\mathrm{~m}, 2 \mathrm{H}), 7.21 \sim 7.24(\mathrm{~m}, 2 \mathrm{H})$, $7.29 \sim 7.32(\mathrm{~m}, 3 \mathrm{H}), 7.37^{*}(\mathrm{~d}, J=5.8 \mathrm{~Hz}, 0.1 \mathrm{H}), 7.43 \sim$ 7.46* (m, 0.3H), 7.48 (dd, $J=4.6,1.3 \mathrm{~Hz}, 1 \mathrm{H}), 7.74 \sim$ 7.76* (m, 0.3H); ${ }^{13} \mathrm{C}$ NMR (75 MHz, $\left.\mathrm{CDCl}_{3}\right) \delta: 55.20$, $55.33,113.55,114.23,121.34,123.15,126.03,127.40$, $128.12,128.68,129.04,129.23,129.64,130.28,131.58$, $132.84,135.88,143.01,146.82,155.94,161.87$.

$N$-[(呋喃-2-基)(苯基)亚甲基]-4-甲氧基苯胺(1b): 黄色固体. m.p. 82.7 84.5 ${ }^{\circ} \mathrm{C} ;{ }^{1} \mathrm{H}$ NMR $(300 \mathrm{MHz}$, $\mathrm{CDCl}_{3}$, 为 $E / Z$ 约 $5 / 1$ 的混合物, 少量组分的核磁数据以 星号进行标记) $\delta: 3.67(\mathrm{~s}, 3 \mathrm{H}), 3.77^{*}(\mathrm{~s}, 0.7 \mathrm{H}), 6.17^{*}(\mathrm{~d}$, $J=3.4 \mathrm{~Hz}, 0.2 \mathrm{H}), 6.32 *(\mathrm{~m}, 0.2 \mathrm{H}), 6.45(\mathrm{~m}, 1 \mathrm{H}), 6.50(\mathrm{~d}$, $J=3.3 \mathrm{~Hz}, 1 \mathrm{H}), 6.62 \sim 6.71(\mathrm{~m}, 4 \mathrm{H}), 6.75 \sim 6.86^{*}(\mathrm{~m}$, $0.9 \mathrm{H}), 7.19 \sim 7.32(\mathrm{~m}, 5 \mathrm{H}), 7.41 \sim 7.47^{*}(\mathrm{~m}, 0.7 \mathrm{H}), 7.61$ $(\mathrm{d}, J=0.8 \mathrm{~Hz}, 1 \mathrm{H}), 7.72 \sim 7.75^{*}(\mathrm{~m}, 0.4 \mathrm{H}) ;{ }^{13} \mathrm{C}$ NMR $(75$ $\left.\mathrm{MHz}, \mathrm{CDCl}_{3}\right) \delta: 55.01,55.20,110.98,111.62,113.42$, $113.98,116.57,116.85,120.37,123.10,127.99,128.79$, $129.05,129.10,130.25,134.89,142.77,143.25,145.35$, 153.43, 155.89, 157.01 .

$N$-[(4-氟苯基)(噻吩-2-基)亚甲基 $]$-4-甲氧基苯胺 (1c): 黄色固体. m.p. $125.3 \sim 127.3{ }^{\circ} \mathrm{C} ;{ }^{1} \mathrm{H}$ NMR $(300$ $\mathrm{MHz}, \mathrm{CDCl}_{3}$, 为 $E / Z$ 约 6/1 的混合物, 少量组分的核磁 数据以星号进行标记): $\delta 3.73(\mathrm{~s}, 3 \mathrm{H}), 3.79^{*}(\mathrm{~s}, 0.5 \mathrm{H})$, 6.71 6.64 (m, $4 \mathrm{H}), 6.74 \sim 6.77 *(\mathrm{~m}, 0.3 \mathrm{H}), 6.81 \sim 6.84 *$ $(\mathrm{m}, 0.3 \mathrm{H}), 6.93 \sim 7.00(\mathrm{~m}, 2 \mathrm{H}), 7.03(\mathrm{~d}, J=3.0 \mathrm{~Hz}, 2 \mathrm{H})$, $7.08 \sim 7.13 *(\mathrm{~m}, 0.3 \mathrm{H}), 7.19 \sim 7.23(\mathrm{~m}, 2 \mathrm{H}), 7.37 \sim 7.38^{*}$ (m, $0.1 \mathrm{H}), 7.38(\mathrm{t}, J=3.1 \mathrm{~Hz}, 1 \mathrm{H}), 7.74 \sim 7.78^{*}(\mathrm{~m}, 0.3 \mathrm{H})$; ${ }^{13} \mathrm{C}$ NMR (75 MHz, $\left.\mathrm{CDCl}_{3}\right) \delta: 55.27,55.37,113.77$, $114.29,114.93,115.18,115.47,121.42,123.05,123.44$, $126.17,127.41,129.48,129.56,130.46,130.49,131.02$, $131.13,131.25,131.36,131.42,131.91,131.96,132.41$, $132.50,136.14,142.99,144.64,146.75,156.16,156.45$, $160.07,160.80,160.98,162.56,164.28,165.88$.

$N$-[(3-氟苯基)(噻吩-2-基)亚甲基]-4-甲氧基苯胺 (1d): 黄色固体. m.p. 106.7 107.9 ${ }^{\circ} \mathrm{C} ;{ }^{1} \mathrm{H}$ NMR (300 $\mathrm{MHz}, \mathrm{CDCl}_{3}$, 为 $E / Z$ 约 $7 / 1$ 的混合物, 少量组分的核磁
数据以星号进行标记) $\delta: 3.73(\mathrm{~s}, 3 \mathrm{H}), 3.80^{*}(\mathrm{~s}, 0.4 \mathrm{H})$, $6.68(\mathrm{~m}, 4 \mathrm{H}), 6.75 \sim 6.79 *(\mathrm{~m}, 0.3 \mathrm{H}), 6.83 \sim 6.86^{*}(\mathrm{~m}$, $0.3 \mathrm{H}), 6.94 \sim 7.06(\mathrm{~m}, 5 \mathrm{H}), 7.18 \sim 7.21 *(\mathrm{~m}, 0.2 \mathrm{H}), 7.25 \sim$ $7.32(\mathrm{~m}, 1 \mathrm{H}), 7.35 \sim 7.43^{*}(\mathrm{~m}, 0.3 \mathrm{H}), 7.47 \sim 7.54(\mathrm{~m}, 1 \mathrm{H})$; ${ }^{13} \mathrm{C}$ NMR $\left(75 \mathrm{MHz}, \mathrm{CDCl}_{3}\right) \delta: 55.19,55.30,113.67$, $114.25,115.65,115.93,115.96,116.25,117.07,117.35$, $121.28,122.95,124.80,124.84,124.96,125.00,126.14$, $127.46,129.52,129.62,129.82,129.93,130.56,131.54$, $132.73,137.91,138.00,142.62,146.16,156.16,160.24$, $160.64,163.92$.

$N$-[(4-氯苯基)(噻吩-2-基)亚甲基]-4-甲氧基苯胺 (1e): 黄色固体. m.p. $158.9 \sim 161.5{ }^{\circ} \mathrm{C} ;{ }^{1} \mathrm{H}$ NMR (300 $\mathrm{MHz}, \mathrm{CDCl}_{3}$, 为 $E / Z$ 约 6/1 的混合物, 少量组分的核磁 数据以星号进行标记) $\delta: 3.72(\mathrm{~s}, 3 \mathrm{H}), 3.79 *(\mathrm{~s}, 0.5 \mathrm{H})$, $6.65 \sim 6.71(\mathrm{~m}, 4 \mathrm{H}), 6.78 \sim 6.82 *(\mathrm{~m}, 0.7 \mathrm{H}), 7.01 \sim 7.04$ (m, 2H), $7.16(\mathrm{~d}, J=8.4 \mathrm{~Hz}, 2 \mathrm{H}), 7.29$ (d, $J=8.4 \mathrm{~Hz}, 2 \mathrm{H})$, $7.39^{*}(\mathrm{~m}, 0.4 \mathrm{H}), 7.48 \sim 7.50(\mathrm{~m}, 1 \mathrm{H}), 7.72 *(\mathrm{~m}, 0.3 \mathrm{H}) ;{ }^{13} \mathrm{C}$ NMR $\left(75 \mathrm{MHz}, \mathrm{CDCl}_{3}\right) \delta: 55.20,55.30,113.71,114.22$, $121.33,122.99,126.15,127.44,128.28,128.47,129.69$, $130.47,130.54,131.44,132.57,134.23,134.69,142.74$, 146.37, 156.10, 160.60 .

$N$-[(4-溴苯基)(噻吩-2-基)亚甲基]-4-甲氧基苯胺 (1f): 黄色固体. m.p. $163.1 \sim 164.5{ }^{\circ} \mathrm{C} ;{ }^{1} \mathrm{H}$ NMR (300 $\mathrm{MHz}, \mathrm{CDCl}_{3}$, 为 $E / Z$ 约 $7 / 1$ 的混合物, 少量组分的核磁 数据以星号进行标记) $\delta: 3.73(\mathrm{~s}, 3 \mathrm{H}), 3.79 *(\mathrm{~s}, 0.4 \mathrm{H})$, $6.71 \sim 6.65(\mathrm{~m}, 4 \mathrm{H}), 6.74 \sim 6.85^{*}(\mathrm{~m}, 0.6 \mathrm{H}), 6.94 \sim 6.99^{*}$ (m, 0.3H), $7.01 \sim 7.04(\mathrm{~m}, 2 \mathrm{H}), 7.09 \sim 7.12(\mathrm{~m}, 2 \mathrm{H}), 7.38^{*}$ (dd, $J=4.9,1.1 \mathrm{~Hz}, 0.2 \mathrm{H}), 7.43 \sim 7.46(\mathrm{~m}, 2 \mathrm{H}), 7.49(\mathrm{q}$, $J=2.0 \mathrm{~Hz}, 1 \mathrm{H}), 7.54 \sim 7.57^{*}(\mathrm{~m}, 0.3 \mathrm{H}), 7.62 \sim 7.65^{*}(\mathrm{~m}$, $0.3 \mathrm{H}) ;{ }^{13} \mathrm{C}$ NMR $\left(75 \mathrm{MHz}, \mathrm{CDCl}_{3}\right) \delta: 55.18,55.28,113.69$, $114.20,121.29,122.97,126.14,127.43,129.73,130.54$, $130.69,130.77,131.23,131.44,132.59 .134 .67,142.68$, $146.27,156.08,160.58$.

$\mathrm{N}$-[(3，5-二溴苯基)(噻吩-2-基)亚甲基]-4-甲氧基苯 胺(1g): 黄色固体. m.p. $116.8 \sim 118.7{ }^{\circ} \mathrm{C} ;{ }^{1} \mathrm{H}$ NMR (300 $\mathrm{MHz}, \mathrm{CDCl}_{3}$, 为 $E / Z$ 约 $4 / 1$ 的混合物, 少量组分的核磁 数据以星号进行标记) $\delta: 3.75(\mathrm{~s}, 3 \mathrm{H}), 3.80^{*}(\mathrm{~s}, 0.9 \mathrm{H})$, $6.67 \sim 6.76(\mathrm{~m}, 5 \mathrm{H}), 6.82 \sim 6.86^{*}(\mathrm{~m}, 0.6 \mathrm{H}), 6.95 \sim 6.97^{*}$ (m, $0.2 \mathrm{H}), 7.98 \sim 7.00(\mathrm{~m}, 1 \mathrm{H}), 7.03 \sim 7.06(\mathrm{~m}, 1 \mathrm{H})$, $7.15 \sim 7.16^{*}(\mathrm{~m}, 0.2 \mathrm{H}), 7.26 \sim 7.28^{*}(\mathrm{~m}, 0.2 \mathrm{H}), 7.31(\mathrm{~d}$, $J=1.7 \mathrm{~Hz}, 2 \mathrm{H}), 7.41 *(\mathrm{dd}, J=4.9,1.1 \mathrm{~Hz}, 0.3 \mathrm{H}), 7.48 \sim$ $7.51 *(\mathrm{~m}, 0.2 \mathrm{H}), 7.52$ (d, $J=0.9 \mathrm{~Hz}, 1 \mathrm{H}), 7.63 *$ (t, $J=1.7$ $\mathrm{Hz}, 0.9 \mathrm{H}), 7.77^{*}$ (t, $\left.J=1.7 \mathrm{~Hz}, 0.2 \mathrm{H}\right), 7.83^{*}(\mathrm{~d}, J=1.7 \mathrm{~Hz}$, $0.4 \mathrm{H}) ;{ }^{13} \mathrm{C}$ NMR $\left(75 \mathrm{MHz}, \mathrm{CDCl}_{3}\right) \delta: 55.28,55.32,113.87$, $114.32,121.22,122.71,122.86,122.94,123.34,126.36$, 
$127.27,127.60,127.69,128.11,130.06,130.38,130.47$, $130.91,130.96,131.64,131.72,132.85,132.95,134.40$, $134.93,135.11,135.54,139.03,139.28,142.01,143.86$, $144.02,145.59,156.39,156.68,158.18,158.54$.

$\mathrm{N}$-[(4-碘苯基)(噻吩-2-基)亚甲基]-4-甲氧基苯胺 (1h): 黄色固体. m.p. 153.0 154.6 ${ }^{\circ} \mathrm{C} ;{ }^{1} \mathrm{H}$ NMR (300 $\mathrm{MHz}, \mathrm{CDCl}_{3}$, 为 $E / Z$ 约 $7 / 1$ 的混合物, 少量组分的核磁 数据以星号进行标记) $\delta: 3.73(\mathrm{~s}, 3 \mathrm{H}), 3.79 *(\mathrm{~s}, 0.4 \mathrm{H})$, 6.64 6.71 (m, 4H), 6.74 6.84* (m, 0.6H), 6.92 6.94* (m, 0.3H), $6.97(\mathrm{~d}, J=8.3 \mathrm{~Hz}, 2 \mathrm{H}), 7.01 \sim 7.04(\mathrm{~m}, 2 \mathrm{H})$, $7.38^{*}(\mathrm{dd}, J=4.9,1.1 \mathrm{~Hz}, 0.1 \mathrm{H}), 7.47 \sim 7.50(\mathrm{~m}, 1 \mathrm{H}), 7.65$ (d, $J=8.3 \mathrm{~Hz}, 2 \mathrm{H}), 7.77 *(\mathrm{~d}, J=8.4 \mathrm{~Hz}, 0.3 \mathrm{H}) ;{ }^{13} \mathrm{C} \mathrm{NMR}$ $\left(75 \mathrm{MHz}, \mathrm{CDCl}_{3}\right) \delta: 55.21,55.30,94.95,113.71,114.22$, $121.28,122.98,126.14,127.44,129.74,130.54,130.78$, $130.85,131.46,132.61,135.27,137.22,137.31,142.69$, 146.26, 156.09, 160.66 .

4-甲氧基- $N$-[(4-硝基苯基)(噻吩-2-基)亚甲基]苯胺 (1i): 黄色固体. m.p. 159.2 160.6 ${ }^{\circ} \mathrm{C} ;{ }^{1} \mathrm{H}$ NMR (300 $\mathrm{MHz}, \mathrm{CDCl}_{3}$, 为 $E / Z$ 约 6/1 的混合物, 少量组分的核磁 数据以星号进行标记) $\delta: 3.72(\mathrm{~s}, 3 \mathrm{H}), 3.81^{*}(\mathrm{~s}, 0.5 \mathrm{H})$, $6.69 \sim 6.62(\mathrm{~m}, 4 \mathrm{H}), 6.78^{*}(\mathrm{~d}, J=8.9 \mathrm{~Hz}, 0.4 \mathrm{H}), 6.86^{*}(\mathrm{~d}$, $J=8.9 \mathrm{~Hz}, 0.4 \mathrm{H}), 6.99 \sim 6.95(\mathrm{~m}, 1 \mathrm{H}), 7.03 \sim 7.05(\mathrm{~m}$, $1 \mathrm{H}), 7.42(\mathrm{~d}, J=8.6 \mathrm{~Hz}, 2 \mathrm{H}), 7.54(\mathrm{~d}, J=4.9 \mathrm{~Hz}, 1 \mathrm{H})$, $7.92 *$ (d, $J=8.7 \mathrm{~Hz}, 0.4 \mathrm{H}), 8.18$ (d, $J=8.7 \mathrm{~Hz}, 2 \mathrm{H}), 8.29 *$ $(\mathrm{d}, J=8.7 \mathrm{~Hz}, 0.3 \mathrm{H}) ;{ }^{13} \mathrm{C} \mathrm{NMR}\left(75 \mathrm{MHz}, \mathrm{CDCl}_{3}\right) \delta: 55.20$, $55.33,113.67,113.84,114.21,114.37,121.22,122.84$, 123.27, 123.44, 126.37, 127.61, 130.10, 130.36, 131.06, $131.54,132.86,142.19,142.63,145.46,147.61156 .41$, 159.55 .

4-甲氧基- $N$-[(噻吩-2-基)(4-三氟甲基苯基)亚甲基] 苯胺 $(\mathbf{1 j})$ : 黄色油状物. ${ }^{1} \mathrm{H} \mathrm{NMR} \mathrm{(300} \mathrm{MHz}, \mathrm{CDCl}_{3}$, 为 $E / Z$ 约 $7 / 1$ 的混合物, 少量组分的核磁数据以星号进行 标记): $\delta 3.73(\mathrm{~s}, 3 \mathrm{H}), 3.81 *(\mathrm{~s}, 0.4 \mathrm{H}), 6.63 \sim 6.70(\mathrm{~m}, 4 \mathrm{H})$, $6.78 *(\mathrm{~d}, J=8.8 \mathrm{~Hz}, 0.3 \mathrm{H}), 6.86^{*}(\mathrm{~d}, J=8.9 \mathrm{~Hz}, 0.3 \mathrm{H})$, $6.97(\mathrm{~d}, J=3.6 \mathrm{~Hz}, 1 \mathrm{H}), 7.02 \sim 7.05(\mathrm{~m}, 1 \mathrm{H}), 7.36$ (d, $J=$ $8.1 \mathrm{~Hz}, 2 \mathrm{H}), 7.52(\mathrm{~d}, J=5.0 \mathrm{~Hz}, 1 \mathrm{H}), 7.58(\mathrm{~d}, J=8.1 \mathrm{~Hz}$, $2 \mathrm{H}), 7.69^{*}(\mathrm{~d}, J=8.5 \mathrm{~Hz}, 0.3 \mathrm{H}), 7.86^{*}(\mathrm{~d}, J=8.0 \mathrm{~Hz}$, $0.4 \mathrm{H}) ;{ }^{13} \mathrm{C}$ NMR $\left(75 \mathrm{MHz}, \mathrm{CDCl}_{3}\right) \delta: 55.14,55.26,76.58$, $77.00,77.20,77.42,113.27,113.74,114.10,114.33$, $118.30,121.18,121.91,122.50,122.95,123.35,124.55$, $124.95,125.00 .125 .08,125.13,125.18,125.23,125.37$, $125.52,126.19,127.08,127.28,127.50,127.83,129.13$, $129.43,129.50,129.79,129.93,130.12,130.36,130.71$, $130.80,131.23,131.55,132.94,135.52,139.59,142.44$, $146.05,156.23,156.62,160.00,160.25$.
4-甲氧基- $N$-[(4-甲氧基苯基)(噻吩-2-基)亚甲基]苯 胺(1k): 黄色油状物. ${ }^{1} \mathrm{H} \mathrm{NMR}\left(300 \mathrm{MHz}, \mathrm{CDCl}_{3}\right.$, 为 $E / Z$ 约 4/1 的混合物, 少量组分的核磁数据以星号进行标记) $\delta: 3.73(\mathrm{~s}, 3 \mathrm{H}), 3.78^{*}(\mathrm{~s}, 0.7 \mathrm{H}), 3.80(\mathrm{~s}, 3 \mathrm{H}), 3.87^{*}(\mathrm{~s}$, $0.6 \mathrm{H}), 6.68 \sim 6.72(\mathrm{~m}, 4 \mathrm{H}), 6.76 \sim 6.79^{*}(\mathrm{~m}, 0.7 \mathrm{H}), 6.80 \sim$ $6.82(\mathrm{~m}, 2 \mathrm{H}), 6.92 \sim 6.97^{*}(\mathrm{~m}, 0.8 \mathrm{H}), 7.00 \sim 7.03(\mathrm{~m}, 1 \mathrm{H})$, $7.02(\mathrm{dd}, J=5.0,3.80 \mathrm{~Hz}, 1 \mathrm{H}), 7.16(\mathrm{dd}, J=9.5,2.0 \mathrm{~Hz}$, $2 \mathrm{H}), 7.35^{*}(\mathrm{dd}, J=5.0,1.0 \mathrm{~Hz}, 0.2 \mathrm{H}), 7.47$ (dd, $J=5.0$, $1.0 \mathrm{~Hz}, 1 \mathrm{H}), 7.73^{*}(\mathrm{~d}, J=8.7 \mathrm{~Hz}, 0.4 \mathrm{H}) ;{ }^{13} \mathrm{C}$ NMR $(75$ $\left.\mathrm{MHz}, \mathrm{CDCl}_{3}\right) \delta: 55.18,55.26,55.34,55.41,113.39$, $113.46,113.65,114.09,121.63,123.16,126.08,127.36$, $127.99,128.82,129.04,130.15,130.78,130.95,131.36$, $132.15,143.37,147.17,155.86,159.70,161.61$.

4-甲氧基- $N$-[(3-甲氧基苯基)(噻吩-2-基)亚甲基]苯 胺(11): 黄色固体. m.p. 109.1 110.2 ${ }^{\circ} \mathrm{C} ;{ }^{1} \mathrm{H}$ NMR (300 $\mathrm{MHz}, \mathrm{CDCl}_{3}$, 为 $E / Z$ 约 $4 / 1$ 的混合物, 少量组分的核磁 数据以星号进行标记) $\delta: 3.70(\mathrm{~s}, 3 \mathrm{H}), 3.72(\mathrm{~s}, 3 \mathrm{H}), 3.80^{*}$ (s, $0.4 \mathrm{H}), 3.85 *(\mathrm{~s}, 0.4 \mathrm{H}), 6.66(\mathrm{~m}, 4 \mathrm{H}), 6.69 \sim 6.72 *(\mathrm{~m}$, $0.3 \mathrm{H}), 6.74 \sim 6.75(\mathrm{~m}, 1 \mathrm{H}), 6.79 \sim 6.85(\mathrm{~m}, 2 \mathrm{H}), 6.87 \sim$ $6.88^{*}(\mathrm{~m}, 0.2 \mathrm{H}), 7.00 \sim 7.03(\mathrm{~m}, 1 \mathrm{H}), 7.07 \sim 7.09(\mathrm{~m}, 1 \mathrm{H})$, $7.19 \sim 7.26(\mathrm{~m}, 1 \mathrm{H}), 7.30 \sim 7.31 *(\mathrm{~m}, 0.2 \mathrm{H}), 7.35 \sim 7.36^{*}$ (m, 0.3H), $7.48(\mathrm{dd}, J=5.0,1.1 \mathrm{~Hz}, 1 \mathrm{H}) ;{ }^{13} \mathrm{C}$ NMR $(75$ $\left.\mathrm{MHz}, \mathrm{CDCl}_{3}\right) \delta: 55.19,55.22,55.33,55.36,113.59$, $113.88,114.24,114.39,114.50,116.59,121.30,121.46$, $122.00,123.00,126.02,127.40,129.00,129.22,129.56$, 130.26, 131.57, 132.77, 137.14, 143.03, 146.64, 156.01, $159.14,161.52$.

$N$-[(苯并 $[d][1,3]$ 二氧杂环戊烷-5-基)(噻吩-2-基)亚 甲基]-4-甲氧基苯胺 (1m): 黄色固体. m.p. 115.3 $117.6{ }^{\circ} \mathrm{C} ;{ }^{1} \mathrm{H} \mathrm{NMR}\left(300 \mathrm{MHz}, \mathrm{CDCl}_{3}\right.$, 为 $E / Z$ 约 $5 / 1$ 的混 合物, 少量组分的核磁数据以星号进行标记) $\delta: 3.74$ (s, $3 \mathrm{H}), 3.78 *(\mathrm{~s}, 0.6 \mathrm{H}), 5.97(\mathrm{~s}, 2 \mathrm{H}), 6.02 *(\mathrm{~s}, 0.4 \mathrm{H}), 6.69(\mathrm{~s}$, $1 \mathrm{H}), 6.70(\mathrm{~m}, 4 \mathrm{H}), 6.73(\mathrm{~d}, J=1.9 \mathrm{~Hz}, 2 \mathrm{H}), 6.76 \sim 6.84 *$ (m, $0.8 \mathrm{H}), 6.89 \sim 6.98^{*}(\mathrm{~m}, 0.7 \mathrm{H}), 7.01 \sim 7.04(\mathrm{~m}, 1 \mathrm{H})$, $7.11(\mathrm{dd}, J=3.7,1.0 \mathrm{~Hz}, 1 \mathrm{H}), 7.14 \sim 7.17 *(\mathrm{~m}, 0.2 \mathrm{H})$, $7.22 \sim 7.25^{*}(\mathrm{~m}, 0.3 \mathrm{H}), 7.35^{*}(\mathrm{~d}, J=1.4 \mathrm{~Hz}, 0.3 \mathrm{H}), 7.36^{*}$ (m, 0.2H), 7.48 (dd, $J=5.0,1.1 \mathrm{~Hz}, 1 \mathrm{H}), 7.64 \sim 7.70 *(\mathrm{~m}$, $0.2 \mathrm{H}) ;{ }^{13} \mathrm{C}$ NMR $\left(75 \mathrm{MHz}, \mathrm{CDCl}_{3}\right) \delta: 55.25,55.32,101.22$, $101.47,101.82$, 107.61, 107.84, 108.10, 109.23, 109.38, $109.60,113.69,114.08,121.58123 .05,123.44,124.56$, $125.46,126.11,127.37,127.77,129.06,129.46,130.27$, $131.39,132.10,133,63,134.10,143.10,146.88,147.32$, $147.84,155.98,161.08$.

4-甲氧基- $N$-[(噻吩-2-基)(4-甲基苯基)亚甲基]-苯胺 (1n): 黄色固体. m.p. $100.1 \sim 101.7{ }^{\circ} \mathrm{C} ;{ }^{1} \mathrm{H}$ NMR (300 
$\mathrm{MHz}, \mathrm{CDCl}_{3}$, 为 $E / Z$ 约 7/1 的混合物, 少量组分的核磁 数据以星号进行标记) $\delta: 2.34(\mathrm{~s}, 3 \mathrm{H}), 2.42 *(\mathrm{~s}, 0.4 \mathrm{H})$, $3.72(\mathrm{~s}, 3 \mathrm{H}), 3.79 *(\mathrm{~s}, 0.4 \mathrm{H}), 6.65 \sim 6.71(\mathrm{~m}, 4 \mathrm{H}), 6.74 \sim$ 6.84* (m, 0.6H), 6.91 6.94* (m, 0.2H), 6.97 6.98* (m, $0.1 \mathrm{H}), 6.99 \sim 7.02(\mathrm{~m}, 1 \mathrm{H}), 7.04 \sim 7.06(\mathrm{~m}, 1 \mathrm{H}), 7.10 \sim$ $7.13(\mathrm{~m}, 4 \mathrm{H}), 7.23 *(\mathrm{~d}, J=8.1 \mathrm{~Hz}, 0.3 \mathrm{H}), 7.36^{*}(\mathrm{~d}, J=4.9$ $\mathrm{Hz}, 0.1 \mathrm{H}), 7.47$ (dd, $J=4.9,1.1 \mathrm{~Hz}, 1 \mathrm{H}), 7.65^{*}(\mathrm{~d}, J=8.1$ $\mathrm{Hz}, 0.3 \mathrm{H}) ;{ }^{13} \mathrm{C} \mathrm{NMR}\left(75 \mathrm{MHz}, \mathrm{CDCl}_{3}\right): \delta 21.32,21.37$, $55.24,55.35,113.64,114.22,121.45,123.12,126.03$, $127.31,128.79,129.06,129.15,129.23,130.10,131.36$, $132.34,132.99,138.61,143.33,147.15,155.97,161.95$.

4-甲氧基- $N$-[(5-甲基噻吩-2-基)(苯基)亚甲基]苯胺 (10): 黄色油状物. ${ }^{1} \mathrm{H} \mathrm{NMR}\left(300 \mathrm{MHz}, \mathrm{CDCl}_{3}\right.$, 为 $E / Z$ 约 $8 / 1$ 的混合物, 少量组分的核磁数据以星号进行标记) $\delta$ : $2.39 *(\mathrm{~s}, 0.36 \mathrm{H}), 2.53(\mathrm{~s}, 3 \mathrm{H}), 3.71(\mathrm{~s}, 3 \mathrm{H}), 3.82^{*}(\mathrm{~s}$, $0.36 \mathrm{H}), 6.57 \sim 6.69(\mathrm{~m}, 5 \mathrm{H}), 6.78 \sim 6.88(\mathrm{~m}, 1.6 \mathrm{H}), 7.18 \sim$ $7.22(\mathrm{~m}, 2 \mathrm{H}), 7.26 \sim 7.30(\mathrm{~m}, 3 \mathrm{H}), 7.42 \sim 7.44^{*}(\mathrm{~m}, 0.4 \mathrm{H})$, $7.71 \sim 7.75^{*}(\mathrm{~m}, 0.2 \mathrm{H}) ;{ }^{13} \mathrm{C}$ NMR $\left(75 \mathrm{MHz}, \mathrm{CDCl}_{3}\right) \delta$ : $15.25^{*}, 15.87,55.22,55.39^{*}, 113.56,114.37,121.23$, $123.17,124.51,125.86,128.03,128.54,129.09,129.28$, $130.02,131.97,133.82,135.90,143.29,144.45,145.61$, 155.86, 161.90 .

4-甲氧基- $N$-[(4-甲基噻吩-2-基)(苯基)亚甲基]苯胺 (1p): 黄色油状物. ${ }^{1} \mathrm{H} \mathrm{NMR} \mathrm{(300} \mathrm{MHz,} \mathrm{CDCl}_{3}$, 为 $E / Z$ 约 $8 / 1$ 的混合物, 少量组分的核磁数据以星号进行标记) $\delta$ : $2.37^{*}(\mathrm{~s}, 0.39 \mathrm{H}), 2.20(\mathrm{~s}, 3 \mathrm{H}), 3.71(\mathrm{~s}, 3 \mathrm{H}), 3.81^{*}(\mathrm{~s}$, $0.36 \mathrm{H}), 6.64 \sim 6.73(\mathrm{~m}, 4 \mathrm{H}), 6.77^{*}$ (d, $J=4.5 \mathrm{~Hz}, 0.25 \mathrm{H}$ ), $6.80 *(\mathrm{~s}, 0.32 \mathrm{H}), 6.84 \sim 6.85(\mathrm{~m}, 1.26 \mathrm{H}), 6.95 *(\mathrm{~s}, 0.15 \mathrm{H})$, $7.07(\mathrm{~s}, 1 \mathrm{H}), 7.20 \sim 7.26(\mathrm{~m}, 2 \mathrm{H}), 7.27 \sim 7.32(\mathrm{~m}, 3 \mathrm{H})$, $7.40 \sim 7.48 *(\mathrm{~m}, 0.5 \mathrm{H}), 7.73 \sim 7.79 *(\mathrm{~m}, 0.3 \mathrm{H}) ;{ }^{13} \mathrm{C} \mathrm{NMR}$ $\left(75 \mathrm{MHz}, \mathrm{CDCl}_{3}\right) \delta: 15.35^{*}, 15.69,55.22,55.37^{*}, 113.58$, $114.27,121.31,122.37,123.16,125.56,125.86,128.08$, $128.24,128.59,129.03,129.23,130.15,133.65,135.04$, 136.09, 137.98, 143.16, 146.41, 155.96, 161.89 .

4-甲氧基- $N$-[(4-甲氧基苯基)(4-硝基苯基)亚甲基] 苯胺 ${ }^{[15]}(\mathbf{1 q})$ : 黄色固体. m.p. $121.4 \sim 122.2{ }^{\circ} \mathrm{C} ;{ }^{1} \mathrm{H}$ NMR $\left(300 \mathrm{MHz}, \mathrm{CDCl}_{3}\right) \delta: 3.72(\mathrm{~s}, 1.8 \mathrm{H}), 3.76(\mathrm{~s}, 1.3 \mathrm{H}), 3.82$ (s, $1.2 \mathrm{H}), 3.86(\mathrm{~s}, 1.7 \mathrm{H}), 6.60(\mathrm{~d}, J=8.8 \mathrm{~Hz}, 1 \mathrm{H}), 6.67 \sim$ $6.76(\mathrm{~m}, 2.8 \mathrm{H}), 6.83(\mathrm{~d}, J=8.7 \mathrm{~Hz}, 1 \mathrm{H}), 6.92(\mathrm{~d}, J=8.8$ $\mathrm{Hz}, 1 \mathrm{H}), 7.03(\mathrm{~d}, J=8.6 \mathrm{~Hz}, 1 \mathrm{H}), 7.31(\mathrm{~d}, J=8.5 \mathrm{~Hz}, 1 \mathrm{H})$, $7.63(\mathrm{~d}, J=8.8 \mathrm{~Hz}, 1 \mathrm{H}), 7.88(\mathrm{~d}, J=8.7 \mathrm{~Hz}, 1 \mathrm{H}), 8.15(\mathrm{~d}$, $J=8.5 \mathrm{~Hz}, 1 \mathrm{H}), 8.23(\mathrm{~d}, J=8.7 \mathrm{~Hz}, 1 \mathrm{H})$.

4-甲氧基- $N$ - $[$ (4-甲氧基苯基)(4-三氟甲基苯基)亚甲 基]苯胺 ${ }^{[5]}$ (1r): 黄色固体. m.p. $115.3 \sim 117.6{ }^{\circ} \mathrm{C} ;{ }^{1} \mathrm{H}$ NMR (300 MHz, $\left.\mathrm{CDCl}_{3}\right) \delta: 3.73 \sim 3.75(\mathrm{~m}, 3.6 \mathrm{H}), 3.81$ (s,
$1.4 \mathrm{H}), 3.86(\mathrm{~s}, 1.8 \mathrm{H}), 3.90(\mathrm{~s}, 1.4 \mathrm{H}), 6.61 \sim 6.76(\mathrm{~m}, 5 \mathrm{H})$, $6.81(\mathrm{~d}, J=8.6 \mathrm{~Hz}, 1 \mathrm{H}), 6.91(\mathrm{~d}, J=8.8 \mathrm{~Hz}, 1 \mathrm{H}), 7.01(\mathrm{q}$, $J=8.6 \mathrm{~Hz}, 2 \mathrm{H}), 7.18 \sim 7.31(\mathrm{~m}, 1.6 \mathrm{H}), 7.54(\mathrm{~d}, J=8.0 \mathrm{~Hz}$, $1 \mathrm{H}), 7.64(\mathrm{~d}, J=7.7 \mathrm{~Hz}, 2 \mathrm{H}), 7.74(\mathrm{~d}, J=8.2 \mathrm{~Hz}, 1 \mathrm{H})$, $7.81 \sim 7.86(\mathrm{~m}, 2.5 \mathrm{H}), 7.94(\mathrm{~d}, J=8.6 \mathrm{~Hz}, 0.3 \mathrm{H}), 8.04(\mathrm{~d}$, $J=8.6 \mathrm{~Hz}, 0.1 \mathrm{H})$.

$\mathrm{N}$-[(4-硝基苯基)(噻吩-2-基)亚甲基]苯胺(1s)：黄色 固体. m.p. 112.4 113.8 ${ }^{\circ} \mathrm{C} ;{ }^{1} \mathrm{H}$ NMR $\left(300 \mathrm{MHz}, \mathrm{CDCl}_{3}\right.$, 为 $E / Z$ 约 $5 / 1$ 的混合物, 少量组分的核磁数据以星号进 行标记) $\delta: 6.71 \sim 6.67(\mathrm{~m}, 2 \mathrm{H}), 6.86 \sim 6.82^{*}(\mathrm{~m}, 0.4 \mathrm{H})$, $7.00 \sim 6.92(\mathrm{~m}, 2.4 \mathrm{H}), 7.06(\mathrm{dd}, J=5.0,3.8 \mathrm{~Hz}, 1 \mathrm{H})$, $7.17 \sim 7.11(\mathrm{~m}, 2.4 \mathrm{H}), 7.36 \sim 7.30 *(\mathrm{~m}, 0.4 \mathrm{H}), 7.43 \sim 7.38$ (m, 2H), 7.57 (dd, $J=5.0,1.3 \mathrm{~Hz}, 1 \mathrm{H}), 7.95^{*}(\mathrm{~d}, J=8.9$ $\mathrm{Hz}, 0.4 \mathrm{H}), 8.16(\mathrm{~d}, J=8.8 \mathrm{~Hz}, 1 \mathrm{H}), 8.30^{*}(\mathrm{~d}, J=8.9 \mathrm{~Hz}$, $0.4 \mathrm{H}) ;{ }^{13} \mathrm{C}$ NMR $\left(75 \mathrm{MHz}, \mathrm{CDCl}_{3}\right) \delta: 119.51,120.65$, $121.09,123.33,124.07,124.30,126.36,127.68,128.69$, $129.23,129.94,130.05,130.24,130.72,131.49,132.01$, $133.28,142.21,145.18,147.74,149.26,160.27$.

4-甲氧基- $N$-[(4-硝基苯基)(噻吩-2-基)亚甲基]苯胺 (1t): 黄色固体. m.p. $142.9 \sim 143.7{ }^{\circ} \mathrm{C} ;{ }^{1} \mathrm{H}$ NMR (300 $\mathrm{MHz}, \mathrm{CDCl}_{3}$, 为 $E / Z$ 约 6/1 的混合物, 少量组分的核磁 数据以星号进行标记) $\delta: 3.66(\mathrm{~s}, 3 \mathrm{H}), 3.71 *(\mathrm{~s}, 0.5 \mathrm{H})$, $6.78 \sim 6.64(\mathrm{~m}, 3 \mathrm{H}), 6.96 \sim 6.89(\mathrm{~m}, 1.5 \mathrm{H}), 6.99(\mathrm{dd}, J=$ $3.7,1.1 \mathrm{~Hz}, 1 \mathrm{H}), 7.05(\mathrm{dd}, J=5.0,3.8 \mathrm{~Hz}, 1 \mathrm{H}), 7.44 \sim 7.36$ $(\mathrm{m}, 2.3 \mathrm{H}), 7.56(\mathrm{dd}, J=5.0,1.1 \mathrm{~Hz}, 1 \mathrm{H}), 8.00 \sim 7.94 *(\mathrm{~m}$, $0.5 \mathrm{H}), 8.13 \sim 8.09(\mathrm{~m}, 2 \mathrm{H}), 8.36 \sim 8.27 *(\mathrm{~m}, 0.5 \mathrm{H}) ;{ }^{13} \mathrm{C}$ NMR (75 MHz, $\left.\mathrm{CDCl}_{3}\right) \delta: 55.14,55.48,111.20,111.60$, $120.17,120.54,121.07,121.46,122.92,123.20,124.95$, $125.19,126.17,127.59,129.31,129.86,130.35,130.40$, $131.42,131.90,132.80,138.91,142.71,144.84,148.77$, 161.86 .

4-氟- $N$-[(4-硝基苯基)(噻吩-2-基)亚甲基]苯胺(1 $\mathbf{u})$ : 黄色固体. m.p. $126.8 \sim 127.6{ }^{\circ} \mathrm{C} ;{ }^{1} \mathrm{H}$ NMR $(300 \mathrm{MHz}$, $\mathrm{CDCl}_{3}$, 为 $E / Z$ 约 $5 / 1$ 的混合物, 少量组分的核磁数据以 星号进行标记) $\delta: 6.67 \sim 6.62(\mathrm{~m}, 2 \mathrm{H}), 6.86 \sim 6.76(\mathrm{~m}$, $2.4 \mathrm{H}), 7.07 \sim 6.97(\mathrm{~m}, 2.6 \mathrm{H}), 7.45 \sim 7.38(\mathrm{~m}, 2.2 \mathrm{H}), 7.57$ (dd, $J=5.0,1.1 \mathrm{~Hz}, 1 \mathrm{H}), 7.94 \sim 7.91 *(\mathrm{~m}, 0.4 \mathrm{H}), 8.19 \sim$ $8.15(\mathrm{~m}, 2 \mathrm{H}), 8.30 \sim 8.27 *(\mathrm{~m}, 0.4 \mathrm{H}) ;{ }^{13} \mathrm{C}$ NMR $(75 \mathrm{MHz}$, $\left.\mathrm{CDCl}_{3}\right) \delta: 115.33,115.62,115.85,116.14,121.06,121.18$, $122.56,122.67,123.32,123.37,123.45,126.48,127.72$, $129.99,130.19,130.75,131.61,132.20,133.29,141.98$, 144.96, 147.75, 157.88, 160.77, 161.10 .

\section{2 .2 二芳基甲酮亚胺 $\mathbf{1 a} \sim 1 \mathrm{u}$ 的不对称氢化硅烷化 反应}

将二芳基甲酮亚胺(1) (0.2 mmol)与催化剂 3c (14.4 
$\mathrm{mg}, 0.04 \mathrm{mmol})$ 溶于 $2 \mathrm{~mL}$ 二氯甲烷, 加入乙酸 $(11 \mu \mathrm{L}$, $0.2 \mathrm{mmol})$, 冷却至 $0{ }^{\circ} \mathrm{C}$, 搅拌下加入三氯硅烷 $(80 \mu \mathrm{L}$, $0.8 \mathrm{mmol}$ )溶于 $320 \mu \mathrm{L}$ 的二氯甲烷的溶液, 继续在 $0{ }^{\circ} \mathrm{C}$ 下搅拌反应至原料消失. 加入饱和碳酸氢钠溶液淬灭反 应, 以乙酸乙酯萃取, 合并有机相, 以无水硫酸钠干燥, 过滤, 减压浓缩, 残余物经硅胶柱层析(石油醚/乙酸乙 酯, $V / V=100 / 1)$ 纯化得目标产物.

4-甲氧基- $N$-[苯基(噻吩-2-基)甲基] 苯胺 (2a) ${ }^{[4 \mathrm{~g}]}$ : 黄 色油状物. 收率 $97 \%, 74 \%$ ee. $[\alpha]_{\mathrm{D}}^{20}-15.0$ (c 0.50 , $\left.\mathrm{CHCl}_{3}\right) ;{ }^{1} \mathrm{H} \mathrm{NMR}\left(300 \mathrm{MHz}, \mathrm{CDCl}_{3}\right) \delta: 3.59$ (s, 3H), 5.81 $(\mathrm{d}, J=7.7 \mathrm{~Hz}, 1 \mathrm{H}), 6.14(\mathrm{~d}, J=7.7 \mathrm{~Hz}, 1 \mathrm{H}), 6.65(\mathrm{~m}, 4 \mathrm{H})$, $6.81(\mathrm{~d}, J=2.9 \mathrm{~Hz}, 1 \mathrm{H}), 6.91 \sim 6.94(\mathrm{~m}, 1 \mathrm{H}), 7.26 \sim 7.22$ (m, 1H), $7.31 \sim 7.39(\mathrm{~m}, 3 \mathrm{H}), 7.47(\mathrm{~d}, J=7.5 \mathrm{~Hz}, 2 \mathrm{H})$. HPLC conditions: Chiralcel OD-H column, $V(\mathrm{EtOH}) /$ $V($ hexane $)=1 / 99$, flow rate $1.0 \mathrm{~mL} / \mathrm{min}$, UV detection at $254 \mathrm{~nm}, t_{\mathrm{R}}($ major $)=9.58 \mathrm{~min}, t_{\mathrm{R}}$ (minor) $=8.97 \mathrm{~min}$.

$N$-[呋喃-2-基(苯基)甲基]-4-甲氧基苯胺 $(\mathbf{2 b})^{[4 \mathrm{~g}]}$ : 黄 色油状物. 收率 $95 \%, 54 \%$ ee. $[\alpha]_{\mathrm{D}}^{20}+5.9$ (c 0.50 , $\left.\mathrm{CHCl}_{3}\right) ;{ }^{1} \mathrm{H} \mathrm{NMR}\left(300 \mathrm{MHz}, \mathrm{CDCl}_{3}\right) \delta: 3.71(\mathrm{~s}, 3 \mathrm{H}), 4.12$ (s, 1H), $5.51(\mathrm{~s}, 1 \mathrm{H}), 6.11(\mathrm{~d}, J=3.1 \mathrm{~Hz}, 1 \mathrm{H}), 6.30-6.31$ (m, 1H), $6.57(\mathrm{~d}, J=8.9 \mathrm{~Hz}, 2 \mathrm{H}), 6.72 \sim 6.75(\mathrm{~m}, 2 \mathrm{H})$, $7.29 \sim 7.43(\mathrm{~m}, 6 \mathrm{H}) ;{ }^{13} \mathrm{C} \mathrm{NMR}\left(75 \mathrm{MHz}, \mathrm{CDCl}_{3}\right) \delta: 55.70$, $57.75,107.32,110.27,114.74,114.91,127.26,127.71$, $128.69,140.74,141.17,142.15,152.47,155.49$. HPLC conditions: Chiralpak AD-H column, $V(i-\mathrm{PrOH}) /$ $V($ hexane $)=1 / 99$, flow rate $1.0 \mathrm{~mL} / \mathrm{min}$, UV detection at $254 \mathrm{~nm}, t_{\mathrm{R}}($ major $)=24.14 \mathrm{~min}, t_{\mathrm{R}}($ minor $)=34.39 \mathrm{mim}$.

$N$-[4-氟苯基(噻吩-2-基)甲基]-4-甲氧基苯胺(2c): 黄色油状物。收率 $95 \%, 74 \% e e .[\alpha]_{\mathrm{D}}^{20}+$ (c 0.50 , $\left.\mathrm{CHCl}_{3}\right) ;{ }^{1} \mathrm{H}$ NMR $\left(300 \mathrm{MHz}, \mathrm{CDCl}_{3}\right) \delta: 3.71(\mathrm{~s}, 3 \mathrm{H}), 3.98$ (s, 1H), $5.84(\mathrm{~s}, 1 \mathrm{H}), 6.46 \sim 6.49(\mathrm{~m}, 2 \mathrm{H}), 6.71 \sim 6.74(\mathrm{~m}$, 2H), $7.23 \sim 7.37(\mathrm{~m}, 8 \mathrm{H}), 7.56(\mathrm{~m}, 1 \mathrm{H}) ;{ }^{3} \mathrm{C}$ NMR $(75$ $\left.\mathrm{MHz}, \mathrm{CDCl}_{3}\right) \delta: 55.67,58.76,114.74,114.98,115.59(\mathrm{~d}$, $J=21.4 \mathrm{~Hz}, 2 \mathrm{C}), 124.97,125.03,128.64(\mathrm{~d}, J=8.1 \mathrm{~Hz}$, 2C), 138.49 (d, $J=3.0 \mathrm{~Hz}, 1 \mathrm{C}), 140.85,147.72,152.58$, 162.16 (d, $J=244.4 \mathrm{~Hz}, 1 \mathrm{C}$ ); HRMS (ESI) calcd for $\mathrm{C}_{18} \mathrm{H}_{16} \mathrm{FNNaOS}[\mathrm{M}+\mathrm{Na}]^{+}$: 336.0829, found 3336.0828. HPLC conditions: Chiralcel OD-H column, $V(i-\mathrm{PrOH}) /$ $V($ hexane $)=1 / 99$, flow rate $1.0 \mathrm{~mL} / \mathrm{min}$, UV detection at $254 \mathrm{~nm}, t_{\mathrm{R}}($ major $)=16.82 \mathrm{~min}, t_{\mathrm{R}}($ minor $)=15.01 \mathrm{~min}$.

$N$-[3-氟苯基(噻吩-2-基)甲基]-4-甲氧基苯胺 (2d): 黄色油状物. 收率 $92 \%, 70 \%$ ee. $[\alpha]_{\mathrm{D}}^{20}+6.0$ (c 0.50 , $\left.\mathrm{CHCl}_{3}\right) ;{ }^{1} \mathrm{H}$ NMR $\left(300 \mathrm{MHz}, \mathrm{CDCl}_{3}\right) \delta: 3.72(\mathrm{~s}, 3 \mathrm{H}), 4.11$ (s, 1H), $5.67(\mathrm{~s}, 1 \mathrm{H}), 6.55(\mathrm{~d}, J=8.8 \mathrm{~Hz}, 2 \mathrm{H}), 6.73(\mathrm{~d}, J=$ $8.8 \mathrm{~Hz}, 2 \mathrm{H}), 6.88(\mathrm{~d}, J=3.4 \mathrm{~Hz}, 1 \mathrm{H}), 6.93 \sim 6.97(\mathrm{~m}, 2 \mathrm{H})$,
$7.15 \sim 7.31(\mathrm{~m}, 3 \mathrm{H}) ;{ }^{13} \mathrm{C}$ NMR $\left(75 \mathrm{MHz}, \mathrm{CDCl}_{3}\right) \delta: 55.69$, 59.00 (d, $J=1.7 \mathrm{~Hz}, 1 \mathrm{C}), 113.93(\mathrm{~d}, J=22.0 \mathrm{~Hz}, 1 \mathrm{C})$, 114.59 (d, $J=21.2 \mathrm{~Hz}, 1 \mathrm{C}), 114.76,114.97,122.64$ (d, $J=$ $2.8 \mathrm{~Hz}, 1 \mathrm{C}$ ), 125.14, 125.17, 126.86, 130.27 (d, $J=8.1 \mathrm{~Hz}$, 1C), 140.76, 145.43 (d, $J=6.4 \mathrm{~Hz}, 1 \mathrm{C}$ ), 147.06, 152.63, 163.13 (d, $J=245.0 \mathrm{~Hz}, 1 \mathrm{C}$ ); HRMS (ESI) calcd for $\mathrm{C}_{18} \mathrm{H}_{15} \mathrm{FNOS}[\mathrm{M}+\mathrm{H}]^{+}: 312.0853$, found 312.0848. HPLC conditions: Chiralpak AD-H column, V(i-PrOH)/ $V($ hexane $)=1 / 99$, flow rate $1.0 \mathrm{~mL} / \mathrm{min}$, UV detection at $254 \mathrm{~nm}, t_{\mathrm{R}}($ major $)=18.28 \mathrm{~min}, t_{\mathrm{R}}($ minor $)=23.71 \mathrm{~min}$.

$\mathrm{N}$-[4-氯苯基(噻吩-2-基)甲基]-4-甲氧基苯胺 (2e): 黄色油状物. 收率 $90 \%, 69 \%$ ee. $[\alpha]_{\mathrm{D}}^{20}-2.7$ (c 0.50 , $\left.\mathrm{CHCl}_{3}\right) ;{ }^{1} \mathrm{H} \mathrm{NMR}\left(300 \mathrm{MHz}, \mathrm{CDCl}_{3}\right) \delta: 3.71(\mathrm{~s}, 3 \mathrm{H}), 4.12$ (s, $1 \mathrm{H}), 5.68(\mathrm{~s}, 1 \mathrm{H}), 6.54 \sim 6.57(\mathrm{~m}, 2 \mathrm{H}), 6.73 \sim 6.76(\mathrm{~m}$, $2 \mathrm{H}), 6.87(\mathrm{~d}, J=3.4 \mathrm{~Hz}, 1 \mathrm{H}), 6.94 \sim 6.96(\mathrm{~m}, 1 \mathrm{H}), 7.24 \sim$ $7.26(\mathrm{~m}, 1 \mathrm{H}), 7.33$ (d, $J=8.5 \mathrm{~Hz}, 2 \mathrm{H}), 7.40(\mathrm{~d}, J=8.5 \mathrm{~Hz}$, $2 \mathrm{H}) ;{ }^{13} \mathrm{C}$ NMR $\left(75 \mathrm{MHz}, \mathrm{CDCl}_{3}\right) \delta: 55.61,58.72,114.67$, $114.91,125.09,125.15,126.84,128.35,128.89,133.27$, $140.67,141.17,147.21,152.51$ ); HRMS (ESI) calcd for $\mathrm{C}_{18} \mathrm{H}_{16} \mathrm{ClNNaOS}[\mathrm{M}+\mathrm{Na}]^{+}:$352.0533, found 352.0520 . HPLC conditions: Chiralpak AD-H column, V(i-PrOH)/ $V($ hexane $)=1 / 99$, flow rate $1.0 \mathrm{~mL} / \mathrm{min}$, UV detection at $254 \mathrm{~nm}, t_{\mathrm{R}}($ major $)=18.42 \mathrm{~min}, t_{\mathrm{R}}($ minor $)=25.96 \mathrm{~min}$.

$\mathrm{N}$-[4-溴苯基(噻吩-2-基)甲基]-4-甲氧基苯胺(2f): 黄 色油状物. 收率 $90 \%, 74 \%$ ee. $[\alpha]_{\mathrm{D}}^{20}-4.4$ ( c 0.50 , $\left.\mathrm{CHCl}_{3}\right) ;{ }^{1} \mathrm{H} \mathrm{NMR}\left(300 \mathrm{MHz}, \mathrm{CDCl}_{3}\right) \delta: 3.72(\mathrm{~s}, 3 \mathrm{H}), 4.10$ $(\mathrm{s}, 1 \mathrm{H}), 5.64(\mathrm{~s}, 1 \mathrm{H}), 6.54(\mathrm{~d}, J=8.9 \mathrm{~Hz}, 2 \mathrm{H}), 6.73(\mathrm{~d}, J=$ $8.9 \mathrm{~Hz}, 2 \mathrm{H}), 6.86(\mathrm{~d}, J=3.4 \mathrm{~Hz}, 1 \mathrm{H}), 6.94 \sim 6.96(\mathrm{~m}, 1 \mathrm{H})$, $7.23 \sim 7.26(\mathrm{~m}, 1 \mathrm{H}), 7.32(\mathrm{~d}, J=8.4 \mathrm{~Hz}, 2 \mathrm{H}), 7.48(\mathrm{~d}, J=$ $8.4 \mathrm{~Hz}, 2 \mathrm{H}) ;{ }^{13} \mathrm{C}$ NMR $\left(75 \mathrm{MHz}, \mathrm{CDCl}_{3}\right) \delta: 55.68,58.89$, $113.82,114.76,115.02,121.47,125.15,125.18,126.86$, $128.75,130.77,131.49,131.88,140.69,141.75,147.13$, 152.65); HRMS (ESI) calcd for $\mathrm{C}_{18} \mathrm{H}_{15} \operatorname{BrNOS}[\mathrm{M}+\mathrm{H}]^{+}$: 372.0052, found 372.0050. HPLC conditions: Chiralpak $\mathrm{AD}-\mathrm{H}$ column, $V(\mathrm{EtOH}) / V($ hexane $)=10 / 90$, flow rate 1.0 $\mathrm{mL} / \mathrm{min}, \mathrm{UV}$ detection at $254 \mathrm{~nm}, t_{\mathrm{R}}$ (major) $=11.08 \mathrm{~min}$, $t_{\mathrm{R}}($ minor $)=12.30 \mathrm{~min}$.

$N$-[3.5-二溴苯基(噻吩-2-基)甲基]-4-甲氧基苯胺 (2g): 黄色油状物. 收率 $92 \%, 68 \% e e .[\alpha]_{\mathrm{D}}^{20}+13.6(\mathrm{c}$ $\left.0.50, \mathrm{CHCl}_{3}\right) ;{ }^{1} \mathrm{H} \mathrm{NMR}\left(300 \mathrm{MHz}, \mathrm{CDCl}_{3}\right) \delta: 3.73(\mathrm{~s}, 3 \mathrm{H})$, $4.12(\mathrm{~s}, 1 \mathrm{H}), 5.59(\mathrm{~s}, 1 \mathrm{H}), 6.51 \sim 6.55(\mathrm{~m}, 2 \mathrm{H}), 6.72 \sim 6.76$ (m, 2H), $6.87(\mathrm{~d}, J=3.3 \mathrm{~Hz}, 1 \mathrm{H}), 6.94 \sim 6.97(\mathrm{~m}, 1 \mathrm{H})$, $7.26 \sim 7.27(\mathrm{~m}, 1 \mathrm{H}), 7.50 \sim 7.58(\mathrm{~m}, 3 \mathrm{H}) ;{ }^{13} \mathrm{C}$ NMR $(75$ $\left.\mathrm{MHz}, \mathrm{CDCl}_{3}\right) \delta: 55.68,58.67,114.84,115.03,123.32$, $125.47,125.57,125.95,126.99,128.37,128.82,130.66$, 
133.37, 140.34, 145.99, 146.85, 152.87); HRMS (ESI) calcd for $\mathrm{C}_{18} \mathrm{H}_{14} \mathrm{Br}_{2} \mathrm{NOS}[\mathrm{M}+\mathrm{H}]^{+}$: 449.9157, found 449.9145. HPLC conditions: Chiralpak AD-H column, $V(i-\mathrm{PrOH}) / V($ hexane $)=1 / 99$, flow rate $1.0 \mathrm{~mL} / \mathrm{min}, \mathrm{UV}$ detection at $254 \mathrm{~nm}, t_{\mathrm{R}}$ (major) $=9.27 \mathrm{~min}, t_{\mathrm{R}}($ minor $)=7.95$ $\min$.

$N$-[4-碘苯基(噻吩-2-基)甲基]-4-甲氧基苯胺(2h): 黄色油状物. 收率 $93 \%, 72 \%$ ee. $[\alpha]_{\mathrm{D}}^{20}-13.1$ (c 0.50 , $\left.\mathrm{CHCl}_{3}\right) ;{ }^{1} \mathrm{H} \mathrm{NMR}\left(300 \mathrm{MHz}, \mathrm{CDCl}_{3}\right) \delta: 3.72$ (s, 3H), 4.10 $(\mathrm{s}, 1 \mathrm{H}), 5.63(\mathrm{~s}, 1 \mathrm{H}), 6.54(\mathrm{~d}, J=8.9 \mathrm{~Hz}, 2 \mathrm{H}), 6.73(\mathrm{~d}, J=$ $8.9 \mathrm{~Hz}, 2 \mathrm{H}), 6.86(\mathrm{~d}, J=3.4 \mathrm{~Hz}, 1 \mathrm{H}), 6.93 \sim 6.95(\mathrm{~m}, 1 \mathrm{H})$, $7.18 \sim 7.26(\mathrm{~m}, 3 \mathrm{H}), 7.68(\mathrm{~d}, J=8.3 \mathrm{~Hz}, 2 \mathrm{H}) ;{ }^{13} \mathrm{C} \mathrm{NMR}$ $\left(75 \mathrm{MHz}, \mathrm{CDCl}_{3}\right) \delta: 55.67,58.96,93.09,114.76,114.99$, $125.12,125.15,126.84,128.99$, 137.82, 140.71, 142.47, 147.10, 152.62); HRMS (ESI) calcd for $\mathrm{C}_{18} \mathrm{H}_{15} \mathrm{INOS}[\mathrm{M}+$ $\mathrm{H}]^{+}$: 419.9914, found 419.9905. HPLC conditions: Chiralpak AD-H column, $V(\mathrm{EtOH}) / V($ hexane $)=10 / 90$, flow rate $1.0 \mathrm{~mL} / \mathrm{min}$, $\mathrm{UV}$ detection at $254 \mathrm{~nm}$, $t_{\mathrm{R}}($ major $)=12.74 \mathrm{~min}, t_{\mathrm{R}}($ minor $)=13.75 \mathrm{~min}$.

4-甲氧基- $N$-[4-硝基苯基(噻吩-2-基)甲基]苯胺(2i): 黄色油状物. 收率 $95 \%, 83 \%$ ee. $[\alpha]_{\mathrm{D}}^{20}+19.8$ (c 0.50 , $\mathrm{CHCl}_{3}$ ); ${ }^{1} \mathrm{H}$ NMR (300 MHz, $\mathrm{CDCl}_{3}$ ) $\delta: 3.71$ (s, 3H), 4.16 $(\mathrm{d}, J=3.6 \mathrm{~Hz}, 1 \mathrm{H}), 5,77(\mathrm{~d}, J=3.9 \mathrm{~Hz}, 1 \mathrm{H}), 6.51(\mathrm{~d}, J=$ $8.9 \mathrm{~Hz}, 2 \mathrm{H}), 6,73(\mathrm{~d}, J=8.9 \mathrm{~Hz}, 2 \mathrm{H}), 6.87 \sim 6.88(\mathrm{~m}, 1 \mathrm{H})$, $6.94 \sim 6.97(\mathrm{~m}, 1 \mathrm{H}), 7.26 \sim 7.29(\mathrm{~m}, 1 \mathrm{H}), 7.65(\mathrm{~d}, J=8.5$ $\mathrm{Hz}, 2 \mathrm{H}), 8.21(\mathrm{~d}, J=8.7 \mathrm{~Hz}, 2 \mathrm{H}) ;{ }^{13} \mathrm{C} \mathrm{NMR}(75 \mathrm{MHz}$, $\left.\mathrm{CDCl}_{3}\right) \delta: 55.63,58.86,114.78,115.02,124.11,125.58$, $125.75,127.00,127.80,140.18,145.64,147.36,150.03$, 152.84); HRMS (ESI) calcd for $\mathrm{C}_{18} \mathrm{H}_{16} \mathrm{~N}_{2} \mathrm{NaO}_{3} \mathrm{~S}[\mathrm{M}+$ $\mathrm{Na}]^{+}:$363.0774, found 363.0762. HPLC conditions: Chiralpak $\mathrm{AD}-\mathrm{H}$ column, $V(\mathrm{EtOH}) / V($ hexane $)=30 / 70$, flow rate $1.0 \mathrm{~mL} / \mathrm{min}$, $\mathrm{UV}$ detection at $254 \mathrm{~nm}, t_{\mathrm{R}}$ (major) $=$ $16.98 \mathrm{~min}, t_{\mathrm{R}}$ (minor) $=19.28 \mathrm{~min}$.

4-甲氧基- $N$-[(噻吩-2-基)(4-三氟甲基苯基)亚甲基] 苯胺 $(\mathbf{2} \mathbf{j})$ : 黄色油状物. 收率 $96 \%, 78 \%$ ee. $[\alpha]_{\mathrm{D}}^{20}+20.2$ (c $0.50, \mathrm{CHCl}_{3}$ ); ${ }^{1} \mathrm{H}$ NMR $\left(300 \mathrm{MHz}, \mathrm{CDCl}_{3}\right) \delta: 3.72$ (s, $3 \mathrm{H}), 4.13(\mathrm{~s}, 1 \mathrm{H}), 5.73$ (s, 1H), 6.53 (d, $J=8.9 \mathrm{~Hz}, 2 \mathrm{H})$, $6.73(\mathrm{~d}, J=8.8 \mathrm{~Hz}, 2 \mathrm{H}), 6.86(\mathrm{~d}, J=3.4 \mathrm{~Hz}, 1 \mathrm{H}), 6.93 \sim$ $6.96(\mathrm{~m}, 1 \mathrm{H}), 7.25 \sim 7.26(\mathrm{~m}, 2 \mathrm{H}), 7.56 \sim 7.63(\mathrm{~m}, 4 \mathrm{H})$; ${ }^{13} \mathrm{C} \mathrm{NMR}\left(75 \mathrm{MHz}, \mathrm{CDCl}_{3}\right) \delta: 55.23,55.63,58.89,59.05$, $113.83,114.79,115.01,115.22,115.41,118.66,122.27$, $123.01,124.84,124.93,125.31,125.35,125.68,125.73$, $125.78,125.83,125.87,126.05,126.60,126.90,127.15$, $127.34,127.53,127.70,129.20,129.48,129.63,130.06$, $130.49,130.74,131.55,137.69,140.58,146.66,146.69$,
152.74); HRMS (ESI) calcd for $\mathrm{C}_{20} \mathrm{H}_{18} \mathrm{ClNNaO}[\mathrm{M}+$ $\mathrm{Na}^{+}$: 386.0797, found 386.0800. HPLC conditions: Chiralpak OJ-H column, $V(\mathrm{EtOH}) / V($ hexane $)=30 / 70$, flow rate $1.0 \mathrm{~mL} / \mathrm{min}$, $\mathrm{UV}$ detection at $254 \mathrm{~nm}, t_{\mathrm{R}}$ (major) $=$ $11.58 \mathrm{~min}, t_{\mathrm{R}}$ (minor) $=10.38 \mathrm{~min}$.

4-甲氧基- $N$-[3-甲氧基苯基(噻吩-2-基)甲基]苯胺 (2l): 黄色油状物. 收率 $94 \%, 75 \%$ ee. $[\alpha]_{\mathrm{D}}^{20}-10.0$ (c 0.50, $\left.\mathrm{CHCl}_{3}\right) ;{ }^{1} \mathrm{H} \mathrm{NMR}\left(300 \mathrm{MHz}, \mathrm{CDCl}_{3}\right) \delta: 3.73(\mathrm{~s}, 3 \mathrm{H})$, $3.79(\mathrm{~s}, 3 \mathrm{H}), 4.16(\mathrm{~s}, 1 \mathrm{H}), 5.66(\mathrm{~s}, 1 \mathrm{H}), 6.58(\mathrm{~d}, J=8.8 \mathrm{~Hz}$, $2 \mathrm{H}), 6.74(\mathrm{~d}, J=8.8 \mathrm{~Hz}, 2 \mathrm{H}), 6.83 \sim 6.85(\mathrm{~m}, 1 \mathrm{H}), 6.91 \sim$ $6.96(\mathrm{~m}, 1 \mathrm{H}), 7.01 \sim 7.06(\mathrm{~m}, 2 \mathrm{H}), 7.22 \sim 7.28(\mathrm{~m}, 2 \mathrm{H})$; ${ }^{13} \mathrm{C}$ NMR $\left(75 \mathrm{MHz}, \mathrm{CDCl}_{3}\right) \delta: 55.15,55.62,59.43,112.66$, $112.86,114.64,114.85,119.29,124.84,126.77,129.75$, $141.05,144.39,147.78,152.38,159.86)$; HRMS (ESI) calcd for $\mathrm{C}_{19} \mathrm{H}_{18} \mathrm{NO}_{2} \mathrm{~S}[\mathrm{M}+\mathrm{H}]^{+}$: 324.1053, found 324.1047. HPLC conditions: Chiralcel OD-H column, $V(i$-PrOH $) / V($ hexane $)=10 / 90$, flow rate $1.0 \mathrm{~mL} / \mathrm{min}, \mathrm{UV}$ detection at $254 \mathrm{~nm}, t_{\mathrm{R}}$ (major) $=7.91 \mathrm{~min}, t_{\mathrm{R}}$ (minor $)=7.15$ $\min$.

$N$-[(苯并 $[\mathrm{d}][1,3]$ 二氧杂环戊烷-5-基)(噻吩-2-基)甲 基]-4-甲氧基苯胺(2m): 黄色油状物. 收率 94\%, $0 \%$ ee. ${ }^{1} \mathrm{H}$ NMR (300 MHz, $\mathrm{CDCl}_{3}$ ) $\delta: 3.72$ (s, 3H), 4.09 (s, 1H), $5.58(\mathrm{~s}, 1 \mathrm{H}), 5.94(\mathrm{~d}, J=0.9 \mathrm{~Hz}, 2 \mathrm{H}), 6.55 \sim 6.58(\mathrm{~m}, 2 \mathrm{H})$, $6.72 \sim 6.81(\mathrm{~m}, 3 \mathrm{H}), 6.88 \sim 6.95(\mathrm{~m}, 4 \mathrm{H}), 7.22(\mathrm{dd}, J=5.0$, $1.1 \mathrm{~Hz}, 1 \mathrm{H}) ;{ }^{13} \mathrm{C} \mathrm{NMR}\left(75 \mathrm{MHz}, \mathrm{CDCl}_{3}\right) \delta: 55.67,59.17$, $101.08,107.38,108.32,114.68,114.89,120.32,124.75$, $124.85,126.80,126.87,136.82,140.98,146.98,147.97$, 148.07, 152.43); HRMS (ESI) calcd for $\mathrm{C}_{19} \mathrm{H}_{17} \mathrm{NNaO}_{3} \mathrm{~S}$ $[\mathrm{M}+\mathrm{Na}]^{+}:$362.0821, found 362.0818. HPLC conditions: Chiralcel OD-H column, $V(\mathrm{EtOH}) / V($ hexane $)=10 / 90$, flow rate $1.0 \mathrm{~mL} / \mathrm{min}$, $\mathrm{UV}$ detection at $254 \mathrm{~nm}, t_{\mathrm{R}}$ (major) $=7.73$ $\min , t_{\mathrm{R}}($ minor $)=8.47 \mathrm{~min}$.

4-甲氧基- $N$-[噻吩-2-基(对甲基苯基)甲基]苯胺(2n): 黄色油状物. 收率 $91 \%, 64 \%$ ee. $[\alpha]_{\mathrm{D}}^{20}-23.3$ (c 0.50 , $\left.\mathrm{CHCl}_{3}\right) ;{ }^{1} \mathrm{H}$ NMR $\left(300 \mathrm{MHz}, \mathrm{CDCl}_{3}\right) \delta: 3.71(\mathrm{~s}, 3 \mathrm{H}), 4.11$ $(\mathrm{s}, 1 \mathrm{H}), 5.65(\mathrm{~s}, 1 \mathrm{H}), 6.55 \sim 6.58(\mathrm{~m}, 2 \mathrm{H}), 6.71 \sim 6.74(\mathrm{~m}$, $2 \mathrm{H}), 6.88 \sim 6.94(\mathrm{~m}, 2 \mathrm{H}), 7.16(\mathrm{~d}, J=7.9 \mathrm{~Hz}, 2 \mathrm{H}), 7.20 \sim$ $7.26(\mathrm{~m}, 1 \mathrm{H}), 7.31(\mathrm{~d}, J=7.9 \mathrm{~Hz}, 2 \mathrm{H}) ;{ }^{13} \mathrm{C} \mathrm{NMR}(75 \mathrm{MHz}$, $\left.\mathrm{CDCl}_{3}\right) \quad \delta: 21.11, \quad 55.69 .59 .25,114.72,114.90 .124 .72$, $126.78,126.93,129.43,137.32,139.86,141.21,148.34$, 152.41); HRMS (ESI) calcd for $\mathrm{C}_{19} \mathrm{H}_{18} \mathrm{NOS}[\mathrm{M}+\mathrm{H}]^{+}$: 308.1104, found 308.1094. HPLC conditions: Chiralpak $\mathrm{AD}-\mathrm{H}$ column, $V(\mathrm{EtOH}) / V($ hexane $)=10 / 90$, flow rate 1.0 $\mathrm{mL} / \mathrm{min}$, UV detection at $254 \mathrm{~nm}, t_{\mathrm{R}}$ (major) $=8.53 \mathrm{~min}$, $t_{\mathrm{R}}($ minor $)=9.63 \mathrm{~min}$. 
4- 甲氧基- $N$-[(5-甲基噻吩-2-基)(苯基)甲基]苯胺 (2o): 黄色油状物. 收率 91\%, 44\% ee. ${ }^{1} \mathrm{H}$ NMR (300 $\left.\mathrm{MHz}, \mathrm{CDCl}_{3}\right) \delta: 2.42(\mathrm{~s}, 3 \mathrm{H}), 3.71(\mathrm{~s}, 3 \mathrm{H}), 4.10(\mathrm{~s}, 1 \mathrm{H})$, $5.59(\mathrm{~s}, 1 \mathrm{H}), 6.54 \sim 6.57(\mathrm{~m}, 3 \mathrm{H}), 6.65(\mathrm{~d}, J=3.3 \mathrm{~Hz}, 1 \mathrm{H})$, $6.70 \sim 6.74(\mathrm{~m}, 2 \mathrm{H}), 7.25 \sim 7.37(\mathrm{~m}, 3 \mathrm{H}), 7.43(\mathrm{~d}, J=7.2$ $\mathrm{Hz}, 2 \mathrm{H}) ;{ }^{13} \mathrm{C}$ NMR $\left(75 \mathrm{MHz}, \mathrm{CDCl}_{3}\right) \delta: 15.36,55.71$, $59.59,114.75,114.86,124.73,126.99,127.54,128.70$, 139.44, 141.22, 142.86, 145.39, 152.39); HRMS (ESI) calcd for $\mathrm{C}_{19} \mathrm{H}_{19} \mathrm{NNaOS}[\mathrm{M}+\mathrm{Na}]^{+}: 332.1080$, found 332.1072. HPLC conditions: Chiralpak AD-H column, $V(i$-PrOH $) / V$ (hexane $)=10 / 90$, flow rate $1.0 \mathrm{~mL} / \mathrm{min}, \mathrm{UV}$ detection at $254 \mathrm{~nm}, t_{\mathrm{R}}$ (major) $=9.99 \mathrm{~min}, t_{\mathrm{R}}($ minor $)=8.01$ $\min$.

4- 甲氧基- $N$-[(4-甲基噻吩-2-基)(苯基)甲基]苯胺 (2p): 黄色油状物. 收率 91\%, 73\% ee. ${ }^{1} \mathrm{H}$ NMR (300 $\left.\mathrm{MHz}, \mathrm{CDCl}_{3}\right) \delta: 2.18(\mathrm{~s}, 3 \mathrm{H}), 3.71(\mathrm{~s}, 3 \mathrm{H}), 4.11(\mathrm{~s}, 1 \mathrm{H})$, $5.60(\mathrm{~s}, 1 \mathrm{H}), 6.53 \sim 6.58(\mathrm{~m}, 2 \mathrm{H}), 6.67(\mathrm{~s}, 1 \mathrm{H}), 6.69 \sim 6.75$ $(\mathrm{m}, 2 \mathrm{H}), 6.79(\mathrm{t}, J=1.1 \mathrm{~Hz}, 1 \mathrm{H}), 7.25 \sim 7.37(\mathrm{~m}, 3 \mathrm{H})$, $7.37 \sim 7.45(\mathrm{~m}, 2 \mathrm{H}) ;{ }^{13} \mathrm{C} \mathrm{NMR}\left(75 \mathrm{MHz}, \mathrm{CDCl}_{3}\right) \delta: 15.78$, $55.71,59.61,114.75,114.89,120.01,127.03,127.23$, $127.59,128.36,128.74,137.45,141.20,142.81,147.73$, 152.44); HRMS (ESI) calcd for $\mathrm{C}_{19} \mathrm{H}_{19} \mathrm{NNaOS}[\mathrm{M}+\mathrm{Na}]^{+}$: 332.1080, found 332.1065. HPLC conditions: Chiralpak AD-H column, $V(i-\mathrm{PrOH}) / V($ hexane $)=10 / 90$, flow rate 1.0 $\mathrm{mL} / \mathrm{min}, \mathrm{UV}$ detection at $254 \mathrm{~nm}, t_{\mathrm{R}}$ (major) $=9.27 \mathrm{~min}$, $t_{\mathrm{R}}(\operatorname{minor})=8.71 \mathrm{~min}$.

4-甲氧基- $N$-[ [4-甲氧基苯基)(4-硝基苯基)甲基]苯 胺(2q): 黄色油状物. 收率 $96 \%, 43 \%$ ee. $[\alpha]_{\mathrm{D}}^{20}+32.2(c$ $\left.0.50, \mathrm{CHCl}_{3}\right) ;{ }^{1} \mathrm{H}$ NMR (300 MHz, $\left.\mathrm{CDCl}_{3}\right) \delta: 3.72(\mathrm{~s}, 3 \mathrm{H})$, $3.5 \sim 4.5$ (brs, 1H), 5.44 (s, 1H), 6.47 (d, $J=8.8 \mathrm{~Hz}, 2 \mathrm{H})$, $6.73(\mathrm{~d}, J=8.8 \mathrm{~Hz}, 2 \mathrm{H}), 6.87(\mathrm{~d}, J=8.6 \mathrm{~Hz}, 2 \mathrm{H}), 7.21$ (d, $J=8.6 \mathrm{~Hz}, 2 \mathrm{H}), 7.59$ (d, $J=8.6 \mathrm{~Hz}, 2 \mathrm{H}), 8.18$ (d, $J=8.6$ $\mathrm{Hz}, 2 \mathrm{H}) ;{ }^{13} \mathrm{C}$ NMR $\left(75 \mathrm{MHz}, \mathrm{CDCl}_{3}\right) \delta: 55.25,55.62$, $62.83,114.33,114.67,114.71,123.96,127.83,128.72$, 134.07, 140.82, 146.98, 150.84, 152.45, 159.23); HRMS (ESI) calcd for $\mathrm{C}_{21} \mathrm{H}_{20} \mathrm{~N}_{2} \mathrm{NaO}_{4}[\mathrm{M}+\mathrm{Na}]^{+}: 387.1315$, found 387.1314.. HPLC.: Chiralpak AD-H column, V(i-PrOH)/ $V($ hexane $)=30 / 70$, flow rate $1.0 \mathrm{~mL} / \mathrm{min}$, UV detection at $254 \mathrm{~nm}, t_{\mathrm{R}}($ major $)=15.40 \mathrm{~min}, t_{\mathrm{R}}($ minor $)=17.10 \mathrm{~min}$.

4- 甲氧基- $N$ - $[$ (4- 甲氧基苯基)(4-三氟甲基苯基)甲 基]苯胺(2r $)^{[5 a]}$ : 黄色油状物. 收率 $95 \%, 38 \%$ ee. $[\alpha]_{\mathrm{D}}^{20}$ +14.0 (c 0.65, $\left.\mathrm{CHCl}_{3}\right) ;{ }^{1} \mathrm{H}$ NMR (300 MHz, $\left.\mathrm{CDCl}_{3}\right) \delta$ : $3.71(\mathrm{~s}, 3 \mathrm{H}), 3.79(\mathrm{~s}, 3 \mathrm{H}), 3.95(\mathrm{~s}, 1 \mathrm{H}), 5.41(\mathrm{~s}, 1 \mathrm{H})$, $6.45 \sim 6.50(\mathrm{~m}, 2 \mathrm{H}), 6.71 \sim 6.73(\mathrm{~m}, 2 \mathrm{H}), 6.86(\mathrm{~d}, J=8.7$ $\mathrm{Hz}, 2 \mathrm{H}), 7.22$ (d, $J=8.6 \mathrm{~Hz}, 2 \mathrm{H}), 7.51$ (d, $J=8.1 \mathrm{~Hz}, 2 \mathrm{H})$, $7.57(\mathrm{~d}, J=8.3 \mathrm{~Hz}, 2 \mathrm{H})$. HPLC conditions: Chiralpak AD-H column, $V(\mathrm{EtOH}) / V($ hexane $)=20 / 80$, flow rate 1.0 $\mathrm{mL} / \mathrm{min}, \mathrm{UV}$ detection at $254 \mathrm{~nm}, t_{\mathrm{R}}$ (major) $=9.35 \mathrm{~min}$, $t_{\mathrm{R}}($ minor $)=10.11 \mathrm{~min}$.

$\mathrm{N}$-[4-硝基苯基(噻吩-2-基)甲基]苯胺(2s)：黄色油状 物. 收率 $87 \%, 89 \%$ ee. $[\alpha]_{\mathrm{D}}^{20}+46.5\left(c \quad 1.30, \mathrm{CHCl}_{3}\right) ;{ }^{1} \mathrm{H}$ NMR (300 MHz, $\left.\mathrm{CDCl}_{3}\right) \delta: 4.43$ (brs, $\left.1 \mathrm{H}\right), 5.87$ (d, $J=5.6$ $\mathrm{Hz}, 1 \mathrm{H}), 6.57$ (d, $J=8.6 \mathrm{~Hz}, 2 \mathrm{H}), 6.78(\mathrm{t}, J=7.5 \mathrm{~Hz}, 1 \mathrm{H})$, $6.90(\mathrm{~d}, J=3.5 \mathrm{~Hz}, 1 \mathrm{H}), 6.97(\mathrm{dd}, J=3.5,1.5 \mathrm{~Hz}, 1 \mathrm{H})$, $7.16(\mathrm{dd}, J=8.5,7.5 \mathrm{~Hz}, 2 \mathrm{H}), 7.30(\mathrm{dd}, J=5.1,1.2 \mathrm{~Hz}$, $1 \mathrm{H}), 7.66(\mathrm{~d}, J=8.6 \mathrm{~Hz}, 2 \mathrm{H}), 8.22(\mathrm{~d}, J=8.7 \mathrm{~Hz}, 2 \mathrm{H}) ;{ }^{13} \mathrm{C}$ NMR $\left(75 \mathrm{MHz}, \mathrm{CDCl}_{3}\right) \delta: 57.94,113.42,113.59,118.69$, $124.08,125.79,125.84,127.02,127.75,129.26,145.26$, $146.03,147.36,149.70$ ); HRMS (ESI) calcd for $\mathrm{C}_{17} \mathrm{H}_{14} \mathrm{~N}_{2} \mathrm{NaO}_{2} \mathrm{~S}[\mathrm{M}+\mathrm{Na}]^{+}$: 333.0668, found 333.0661. HPLC conditions: Chiralpak AD-H column, $V(\mathrm{EtOH})$ $V($ hexane $)=10 / 90$, flow rate $1.0 \mathrm{~mL} / \mathrm{min}, \mathrm{UV}$ detection at $254 \mathrm{~nm}, t_{\mathrm{R}}($ major $)=16.34 \mathrm{~min}, t_{\mathrm{R}}($ minor $)=17.85 \mathrm{~min}$.

4-甲氧基- $\mathrm{N}$-[4-硝基苯基(噻吩-2-基)甲基]苯胺(2t): 黄色油状物. 收率 98\%, 79\% ee. $[\alpha]_{\mathrm{D}}^{20}+38.1$ (c 1.10, $\left.\mathrm{CHCl}_{3}\right) ;{ }^{1} \mathrm{H}$ NMR $\left(300 \mathrm{MHz}, \mathrm{CDCl}_{3}\right) \delta: 3.88(\mathrm{~s}, 3 \mathrm{H}), 5.01$ (s, $1 \mathrm{H}), 5.84(\mathrm{~d}, J=1.7 \mathrm{~Hz}, 1 \mathrm{H}), 6.36(\mathrm{~d}, J=9.3 \mathrm{~Hz}, 1 \mathrm{H})$, $6.75 \sim 6.72(\mathrm{~m}, 1 \mathrm{H}), 6.82 \sim 6.80(\mathrm{~m}, 1 \mathrm{H}), 6.88(\mathrm{~d}, J=3.5$ $\mathrm{Hz}, 1 \mathrm{H}), 6.97$ (dd, $J=5.0,3.6 \mathrm{~Hz}, 1 \mathrm{H}), 7.30 \sim 7.26(\mathrm{~m}$, $1 \mathrm{H}), 7.65(\mathrm{~d}, J=8.7 \mathrm{~Hz}, 2 \mathrm{H}), 8.22(\mathrm{~d}, J=8.7 \mathrm{~Hz}, 2 \mathrm{H}) ;{ }^{13} \mathrm{C}$ NMR (75 MHz, $\left.\mathrm{CDCl}_{3}\right) \delta: 55.45,57.88,58.08,109.46$, $109.57,110.98,111.12,117.62,117.96,121.01,122.89$, 124.04, 124.09, 125.71, 125.74, 126.71, 127.00, 127.82, $136.05,145.56,146.88,147.40,149.93$ ); HRMS (ESI) calcd for $\mathrm{C}_{18} \mathrm{H}_{16} \mathrm{~N}_{2} \mathrm{NaO}_{3} \mathrm{~S}[\mathrm{M}+\mathrm{Na}]^{+}: 363.0774$, found 363.0767. HPLC conditions: Chiralpak AD-H column, $V(\mathrm{EtOH}): V(i-\mathrm{PrOH}): V($ hexane $)=10: 5: 85$, flow rate $1.0 \mathrm{~mL} / \mathrm{min}$, UV detection at $254 \mathrm{~nm}, t_{\mathrm{R}}$ (major) $=8.37$ $\min , t_{\mathrm{R}}(\operatorname{minor})=8.86 \mathrm{~min}$.

4-氟- $N$-[4-硝基苯基(噻吩-2-基)甲基]苯胺 $(2 \mathbf{u})$ : 黄 色油状物. 收率 $93 \%, 89 \%$ ee. $[\alpha]_{\mathrm{D}}^{20}+53.9$ (c 0.94 , $\left.\mathrm{CHCl}_{3}\right) ;{ }^{1} \mathrm{H}$ NMR $\left(300 \mathrm{MHz}, \mathrm{CDCl}_{3}\right) \delta: 4.33(\mathrm{~s}, 1 \mathrm{H}), 5.80$ (s, 1H), 6.50 (dd, $J=9.0,4.4 \mathrm{~Hz}, 2 \mathrm{H}), 6.96$ (dd, $J=5.1,1.5$ $\mathrm{Hz}, 1 \mathrm{H}), 7.29$ (dd, $J=5.1,1.2 \mathrm{~Hz}, 1 \mathrm{H}), 7.64$ (d, $J=8.5 \mathrm{~Hz}$, $2 \mathrm{H}), 8.22(\mathrm{~d}, J=8.8 \mathrm{~Hz}, 2 \mathrm{H}) ;{ }^{13} \mathrm{C}$ NMR $\left(75 \mathrm{MHz}, \mathrm{CDCl}_{3}\right)$ $\delta: 58.52,114.59$ (d, $J=7.4 \mathrm{~Hz}, 2 \mathrm{C}), 115.73$ (d, $J=22.4 \mathrm{~Hz}$, 2C), 124.12, 125.74, 125.86, 127.05, 127.75, 142.37 (d, $J=2.0 \mathrm{~Hz}, 1 \mathrm{C}), 145.16,147.40,149.52,156.32$ (d, $J=$ $235.5 \mathrm{~Hz}, 1 \mathrm{C}$ ); HRMS (ESI) calcd for $\mathrm{C}_{17} \mathrm{H}_{13} \mathrm{FN}_{2} \mathrm{NaO}_{3} \mathrm{~S}$ $[\mathrm{M}+\mathrm{Na}]^{+}:$351.0574, found 351.0573. HPLC conditions: 
Chiralpak AD-H column, $V(\mathrm{EtOH}) / V($ hexane $)=15 / 85$, flow rate $1.0 \mathrm{~mL} / \mathrm{min}$, $\mathrm{UV}$ detection at $254 \mathrm{~nm}$, $t_{\mathrm{R}}($ major $)=14.98 \mathrm{~min}, t_{\mathrm{R}}($ minor $)=16.67 \mathrm{~min}$.

\section{辅助材料(Supporting Information) 化合物 1 和 3 的 ${ }^{1} \mathrm{H}$} $\mathrm{NMR} 、{ }^{13} \mathrm{C} N M R$ 谱图以及化合物 $\mathbf{3}$ 的手性 HPLC 谱图. 这些材料可以免费从本刊网站(http://sioc-journal.cn/)上 下载.

\section{References}

[1] (a) Spencer, C. M.; Faulds, D.; Peters, D. H. Drugs 1993, 46, 1055. (b) Bishop, M. J.; Mcnutt, R. W. Bioorg. Med. Chem. Lett. 1995, 5, 1311.

(c) Bilsky, E. J.; Calderon, S. N.; Wang, T.; Bernstein, R. N.; Davis, P.; Hruby, V. J.; Menutt, R. W.; Rothman, R. B.; Rice, K. C.; Porreca, F. J. Pharmacol. Exp. Ther. 1995, 273, 359.

(d) Sakurai, S.; Ogawa, N.; Suzuki, T.; Kato, K.; Ohashi, T.; Yasuda, S.; Kato, H.; Ito, Y. Chem. Pharm. Bull. 1996, 44, 765.

(e) Plobeck, N.; Delorme, D.; Wei, Z. Y.; Yang, H.; Zhou, F.; Schwarz, P.; Gawell, L.; Gagnon, H.; Pelcman, B.; Schmidt, R.; Yue, S. Y.; Walpole, C.; Brown, W.; Zhou, E.; Labarre, M.; Payza, K.; St-Onge, S.; Kamassah, A.; Morin, P. E.; Projean, D.; Ducharme, J.; Roberts, E. J. Med.Chem. 2000, 43, 3878.

(f) Gillard, M.; Van Der Perren, C.; Moguilevsky, N.; Massingham, R.; Chatelain, P. Mol. Pharmacol. 2002, 61, 391.

(g) Cetinkaya, Y.; Gocer, H.; Goksu, S.; Gulcin, I. J. Enzyme Inhib. Med. Chem. 2014, 29, 168.

[2] For a review, see: Schmidt, F.; Stemmler, R. T.; Rudolph, J.; Bolm, C. Chem. Soc. Rev. 2006, 35, 454.

[3] (a) Tomioka, K.; Inoue, I.; Shindo, M.; Koga, K. Tetrahedron Lett. 1990, 31, 6681 .

(b) Delorme, D.; Berthelette, C.; Lavoie, R.; Roberts, E. Tetrahedron: Asymmetry 1998, 9, 3963.

(c) Pflum, D. A.; Krishnamurthy, D.; Han, Z. X.; Wald, S. A.; Senanayake, C. H. Tetrahedron Lett. 2002, 43, 923.

(d) Plobeck, N.; Powell, D. Tetrahedron: Asymmetry 2002, 13, 303.

(e) Han, Z. X.; Krishnamurthy, D.; Grover, P.; Fang, Q. K.; Pflum, D. A.; Senanayake, C. H. Tetrahedron Lett. 2003, 44, 4195.

(f) Cabello, N.; Kizirian, J. C.; Alexakis, A. Tetrahedron Lett. 2004, 45,4639 .

(g) Weix, D. J.; Shi, Y. L.; Ellman, J. A. J. Am. Chem. Soc. 2005, 127, 1092.

(h) Bolshan, Y.; Batey, R. A. Org. Lett. 2005, 7, 1481.

(i) Boebel, T. A.; Hartwig, J. F. Tetrahedron 2008, 64, 6824.

(j) Haurena, C.; LeGall, E.; Sengmany, S.; Martens, T. Tetrahedron 2010, 66, 9902 .

(k) Han, Z. X.; Busch, R.; Fandrick, K. R.; Meyer, A.; Xu, Y. B.; Krishnamurthy, D. K.; Senanayake, C. H. Tetrahedron 2011, 67, 7035 .

[4] (a) Hayashi, T.; Ishigedani, M. J. Am. Chem. Soc. 2000, 122, 976.

(b) Hermanns, N.; Dahmen, S.; Bolm, C.; Brase, S. Angew. Chem., Int. Ed. 2002, 41, 3692.

(c) Kuriyama, M.; Soeta, T.; Hao, X. Y.; Chen, O.; Tomioka, K. J. Am. Chem. Soc. 2004, 126, 8128.

(d) Tokunaga, N.; Otomaru, Y.; Okamoto, K.; Ueyama, K.; Shintani, R.; Hayashi, T. J. Am. Chem. Soc. 2004, 126, 13584.

(e) Hayashi, T.; Kawai, M.; Tokunaga, N. Angew. Chem., Int. Ed. 2004, 43,6125 . (f) Otomaru, Y.; Tokunaga, N.; Shintani, R.; Hayashi, T. Org. Lett. 2005, 7, 307.

(g) Cabello, N.; Kizirian, J. C.; Gille, S.; Alexakis, A.; Bernardinelli, G.; Pinchard, L.; Caille, J. C. Eur. J. Org. Chem. 2005, 4835 .

(h) Jagt, R. B. C.; Toullec, P. Y.; Geerdink, D.; de Vries, J. G.; Feringa, B. L.; Minnaard, A. D. J. Angew. Chem., Int. Ed. 2006, 45, 2789.

(i) Duan, H. F.; Jia, Y. X.; Wang, L. X.; Zhou, Q. L. Org. Lett. 2006, 8, 2567-2569.

(j) Wang, Z. Q.; Feng, C. G.; Xu, M. H.; Lin, G. Q. J. Am. Chem. Soc. 2007, 129, 5336.

(k) Clayden, J.; Dufour, J.; Grainger, D. M.; Helliwell, M. J. Am. Chem. Soc. 2007, 129, 7488.

(1) Nakagawa, H.; Rech, J. C.; Sindelar, R. W.; Ellman, J. A. Org. Lett. 2007, 9, 5155.

(m) Marelli, C.; Monti, C.; Gennari, C.; Piarulli, U. Synlett 2007, 2213.

(n) Okamoto, K.; Hayashi, T.; Rawal, V. H. Chem. Commun. 2009, 4815.

(o) Hao, X. Y.; Kuriyama, M.; Chen, Q.; Yamamoto, Y.; Yamada, K.; Tomioka, K. Org. Lett. 2009, 11, 4470.

(p) Shintani, R.; Takeda, M.; Tsuji, T.; Hayashi, T. J. Am. Chem. Soc. 2010, 132, 13168.

(q) Cao, Z. P.; Du, H. F. Org. Lett. 2010, 12, 2602.

(r) Shintani, R.; Soh, Y. T.; Hayashi, T. Org. Lett. 2010, 12, 4106.

(s) Shao, C.; Yu, H. J.; Wu, N. Y.; Feng, C. G.; Lin, G. Q. Org. Lett. 2010, 12, 3820.

(t) Wang, L.; Wang, Z. Q.; Xu, M. H.; Lin, G. Q. Synthesis 2010, 3263.

(u) Crampton, R.; Woodward, S.; Fox, M. Adv. Synth. Catal. 2011, 353, 903.

(v) Hao, X. Y.; Chen, Q.; Kuriyama, M.; Yamada, K.; Yamamoto, Y.; Tomioka, K. Catal. Sci. Technol. 2011, 1, 62.

(w) Shintani, R.; Narui, R.; Tsutsumi, Y.; Hayashi, S.; Hayashi, T. Chem. Commun. 2011, 47, 6123.

(x) Han, W. Y.; Wu, Z. J.; Zhang, X. M.; Yuan, W. C. Org. Lett. 2012, 14, 976.

(y) Han, W. Y.; Zuo, J.; Zhang, X. M.; Yuan, W. C. Tetrahedron 2013, 69, 537.

[5] (a) Malkov, A. V.; Vrankova, K.; Stončius, S.; Kočovsky, P. J. Org. Chem. 2009, 74, 5839.

(b) Hou, G. H.; Tao, R.; Sun, Y.; Zhang, X. M.; Gosselin, F. J. Am. Chem. Soc. 2010, 132, 2124.

(c) Nguyen, T. B.; Wang, Q.; Gueritte, F. Chem. Eur. J. 2011, 17, 9576.

(d) Thanh, B. N.; Bousserouel, H.; Wang, Q. A.; Gueritte, F. $A d v$. Synth. Catal. 2011, 353, 257.

(e) Amezquita-Valencia, M.; Cabrera, A. J. Mol. Catal. A: Chem. 2013, 366, 17.

(f) Hu, X. Y.; Zhang, M. M.; Shu, C.; Zhang, Y. H.; Liao, L. H.; Yuan, W. C.; Zhang, X. M. Adv. Synth. Catal. 2014, 356, 3539.

[6] Chu, L.; Wang, X. C.; Moore, C. E.; Rheingold, A. L.; Yu, J. Q. J. Am. Chem. Soc. 2013, 135, 16344.

[7] For some recent reviews, see: (a) Zhou, Q. L.; Xie, J. H. Top. Curr. Chem. 2014, 343, 75.

(b) Li, X. H.; Wu, P. Curr. Org. Chem. 2014, 18, 1242-1261.

(c) Ji, Y. G.; Wu, L.; Fan, Q. H. Acta Chim. Sinica 2014, 72, 798 (in Chinese).

(季益刚, 吴否, 范青华, 化学学报, 2014, 72, 798.)

(d) He, Y. M.; Song, F. T.; Fan, Q. H. Top. Curr. Chem. 2014, 343, 145. 
(e) Wang, D.; Hou, C. J.; Chen, L. F.; Liu, X. N.; An, Q. D.; Hu, X. P. Chin. J. Org. Chem. 2013, 33, 1355 (in Chinese).

(王东, 侯传金, 陈丽风, 刘小宁, 安庆大, 胡向平, 有机化学, 2013, 33, 1355.)

(f) Chen, Q. A.; Ye, Z. S.; Duan, Y.; Zhou, Y. G. Chem. Soc. Rev. 2013, 42, 497.

(g) Etayo, P.; Vidal-Ferran, A. Chem. Soc. Rev. 2013, 42, 728.

(h) Xie, J. H.; Zhu, S. F.; Zhou, Q. L. Chem. Soc. Rev. 2012, 41, 4126.

(i) Xie, J. H.; Zhou, Q. L. Acta Chim. Sinica 2012, 70, 1427 (in Chinese).

(谢建华, 周其林, 化学学报, 2012, 70, 1427.)

(j) Nugent, T. C.; El-Shazly, M. Adv. Synth. Catal. 2010, 352, 753.

(k) Cheng, T. Y.; Zhao, Q. K.; Zhang, D. C.; Liu, G. H. Curr. Org. Chem. 2015, 19, 667.

(1) Foubelo, F.; Yus, M. Chem. Rec. 2015, 15, 907.

(m) Zheng, C.; You, S. L. Chem. Soc. Rev. 2012, 41, 2498.

(n) Robertson, A.; Matsumoto, T.; Ogo, S. Dalton Trans. 2011, 40, 10304.

(o) Tang, Y. F.; Deng, J. G. Prog. Chem. 2010, 22, 1242.

(p) Wang, Z. Y.; Jiang, Z. J. Asian J. Chem. 2010, 22, 4141.

(q) Jones, S.; Warner, C. J. A. Org. Biomol. Chem. 2012, 10, 2189. (r) Weickgenannt, A.; Oestreich, M. Chem CatChem 2011, 3, 1527. (s) Guizzetti, S.; Benaglia, M. Eur. J. Org. Chem. 2010, 5529.

[8] Zheng, H. J.; Deng, J. G.; Lin, W. Q.; Zhang, X. M. Tetrahedron Lett. 2007, 48, 7934.

[9] Jiang, Y.; Chen, X.; Zheng, Y. S.; Xue, Z. Y.; Shu, C.; Yuan, W. C.; Zhang, X. M. Angew. Chem., Int. Ed. 2011, 50, 7304.

[10] Chen, X.; Hu, X. Y.; Shu, C.; Zhang, Y. H.; Zheng, Y. S.; Jiang, Y.; Yuan, W. C.; Liu, B.; Zhang, X. M. Org. Biomol. Chem. 2013, 11, 3089.

[11] Xue, Z. Y.; Jiang, Y.; Yuan, W. C.; Zhang, X. M. Eur. J. Org. Chem. 2010, 616.

[12] Zheng, H. J.; Deng, J. G.; Lin, W. Q.; Zhang, X. M. Tetrahedron Lett. 2007, 48, 7934.

[13] CCDC 1038813 (for 2a) contains the supplementary crystallo graphic data for this paper. These data can be obtained free of charge from The Cambridge Crystallographic Data Centre via www. ccdc. cam. ac. uk/data_request/cif.

[14] Artico, M.; De Martino, G.; Pasquali, A. D.; Regina, G. L.; Silvestri, R.; Ragno, R.; Bergamini, A.; Ciaprini, C.; Sinistro, A.; Crespan, E.; Maga, G. J. Med. Chem. 2003, 46, 2482.

[15] Sato, M.; Nagashima, S.; Murakami, M.; Kaneko, C. Tetrahedron 1993, 49, 6575 .

(Li, L.; Fan, Y.) 